\title{
Carbon Monoxide Modulation of Microglia-Neuron Communication: Anti-Neuroinflammatory and Neurotrophic Role
}

\author{
Nuno L. Soares ${ }^{1} \cdot$ Inês Paiva ${ }^{1,2}$. Joana Bravo ${ }^{3}$. Cláudia S. F. Queiroga ${ }^{1}$ - Bernadete F. Melo ${ }^{1}$. Sílvia V. Conde ${ }^{1}$. \\ Carlos C. Romão ${ }^{4}$ Teresa Summavielle ${ }^{3} \cdot$ Helena L. A. Vieira ${ }^{1,2,5,6}$
}

Received: 16 September 2021 / Accepted: 9 November 2021

(c) The Author(s), under exclusive licence to Springer Science+Business Media, LLC, part of Springer Nature 2021

\begin{abstract}
Microglia, the 'resident immunocompetent cells' of the central nervous system (CNS), are key players in innate immunity, synaptic refinement and homeostasis. Dysfunctional microglia contribute heavily to creating a toxic inflammatory milieu, a driving factor in the pathophysiology of several CNS disorders. Therefore, strategies to modulate the microglial function are required to tackle exacerbated tissue inflammation. Carbon monoxide (CO), an endogenous gaseous molecule produced by the degradation of haem, has anti-inflammatory, anti-apoptotic, and pro-homeostatic and cytoprotective roles, among others. ALF-826A, a novel molybdenum-based CO-releasing molecule, was used for the assessment of neuron-microglia remote communication. Primary cultures of rat microglia and neurons, or the BV-2 microglial and CAD neuronal murine cell lines, were used to study the microglia-neuron interaction. An approach based on microglial-derived conditioned media in neuronal culture was applied. Medium derived from CO-treated microglia provided indirect neuroprotection against inflammation by limiting the lipopolysaccharide (LPS)-induced expression of reactivity markers (CD11b), the production of reactive oxygen species (ROS) and the secretion of inflammatory factors (TNF- $\alpha$, nitrites). This consequently prevented neuronal cell death and maintained neuronal morphology. In contrast, in the absence of inflammatory stimulus, conditioned media from COtreated microglia improved neuronal morphological complexity, which is an indirect manner of assessing neuronal function. Likewise, the microglial medium also prevented neuronal cell death induced by pro-oxidant tert-Butyl hydroperoxide $(t$-BHP). ALF-826 treatment reinforced microglia secretion of Interleukin-10 (IL-10) and adenosine, mediators that may protect against $t$-BHP stress in this remote communication model. Chemical inhibition of the adenosine receptors $\mathrm{A}_{2 \mathrm{~A}}$ and $\mathrm{A}_{1}$ reverted the $\mathrm{CO}$-derived neuroprotective effect, further highlighting a role for $\mathrm{CO}$ in regulating neuron-microglia communication via purinergic signalling. Our findings indicate that $\mathrm{CO}$ has a modulatory role on microglia-to-neuron communication, promoting neuroprotection in a non-cell autonomous manner. $\mathrm{CO}$ enhances the microglial release of neurotrophic factors and blocks exacerbated microglial inflammation. $\mathrm{CO}$ improvement of microglial neurotrophism under non-inflammatory conditions is here described for the first time.
\end{abstract}

Keywords Microglia $\cdot$ Neurons $\cdot$ Carbon monoxide $\cdot$ Neuroinflammation $\cdot$ Neurotrophism

Helena L. A. Vieira

hl.vieira@fct.unl.pt

1 CEDOC-Faculdade de Ciências Médicas/NOVA Medical School, Universidade Nova de Lisboa, Lisbon, Portugal

2 UCIBIO, Applied Molecular Biosciences Unit, Department of Chemistry, NOVA School of Science and Technology, Universidade Nova de Lisboa, Lisbon, Portugal

3 i3S-Instituto de Investigação E Inovação Em Saúde and IBMC-Instituto de Biologia Molecular E Celular, Universidade Do Porto, 4200-135 Porto, Portugal
4 Instituto de Tecnologia Química E Biológica António Xavier, Universidade Nova de Lisboa, Oeiras, Portugal

5 Associate Laboratory i4HB-Institute for Health and Bioeconomy, NOVA School of Science and Technology, Universidade Nova de Lisboa, Lisbon, Portugal

6 UCIBIO, Faculdade de Ciências E Tecnologia, Universidade NOVA de Lisboa, Campus de Caparica, 2829-526 Caparica, Portugal 


\begin{tabular}{ll}
\multicolumn{2}{l}{ Abbreviations } \\
BDNF & Brain-derived neurotrophic factor \\
CO & Carbon monoxide \\
CORM & Carbon monoxide-releasing molecules \\
CNS & Central nervous system \\
DPCPX & Dipropylcyclopentylxanthine \\
H2DCFDA & $2^{\prime}, 7^{\prime}$-Dichlorofluorescein diacetate \\
ERK & Extracellular-signal-regulated kinase \\
FBS & Foetal bovine serum \\
GDNF & Glial cell-derived neurotrophic factor \\
HO & Haem oxygenase \\
IGF-1 & Insulin growth factor 1 \\
IFN- $\gamma$ & Interferon $\gamma$ induced \\
IL-1 $\beta$ & Interleukin-1 $\beta$ \\
IL-10 & Interleukin-10 \\
LPS & Lipopolysaccharide \\
LRP3 & NLR family pyrin domain-containing 3 \\
NO & Nitric oxide \\
PFA & Paraformaldehyde \\
PDL & Poly-D-lysine \\
PPAR- $\gamma$ & Peroxisome proliferator-activated receptor \\
& gamma co-activator 1 $\alpha$ \\
PI3K & Phosphoinositide 3-kinase \\
ROS & Reactive oxygen species \\
RT & Room temperature \\
SDS-PAGE & Sodium dodecyl sulphate-polyacrylamide \\
$t$-BHP & gel electrophoresis \\
TNF- $\alpha$ & tert-Butyl hydroperoxide \\
& Tumour necrosis factor $\alpha$ \\
&
\end{tabular}

\section{Background}

Microglia are the main immune cell population in the central nervous system (CNS) [1]. Originating from a primitive myeloid precursor [2], microglia populate the brain and spinal cord, where they account for $5-10 \%$ of total glial population with crucial biological functions [1, 3]. As the brain first line of defence, microglia are sentinels that constantly screen the brain parenchyma. When encountering potentially pathogenic or toxic agents that endanger the CNS integrity, microglia initiate an inflammatory response $[1,3]$. This response involves major functional and morphological changes, namely alteration of microglia expression of surface receptors, intracellular enzymes and secreted molecules [1]. Release of inflammatory mediators, such as reactive oxygen species (ROS) $[4,5]$ and nitric oxide (NO) derivatives $[3,6,7]$, cytokines $[3,6,7]$, metabolites and extracellular vesicles [8] induce cell death on compromised cellular populations and/or potentiate the microglial phagocytosis of bacteria, viruses and debris $[1,9,10]$. Additionally, microglia recruit other cells and help set up the adaptive immune response [11]. Conversely, microglia also have a relevant role in neurotrophic support [12], as well as brain development, removing immature and apoptotic populations [13]. Microglia also participate in the regulation of synaptic plasticity and neuronal activity [14-17], and stimulate synaptogenesis and synaptic maturation $[18,19]$. Several of these functions are reliant on microglia paracrine mechanisms, through secretion of specific factors like thrombospondin, insulin growth factor 1 (IGF-1) [12, 20-24], brain-derived neurotrophic factor (BDNF), adenosine, interleukin-10 (IL$10)$ as well as low levels of inflammatory cytokines TNF- $\alpha$ (tumour necrosis factor $\alpha$ ) and IL-1 $\beta$ [25-28]. Being both the hub of neuroinflammation and crucial for neuronal development, survival and activity, microglia function is under tight regulation. Deficient microglial function causes imbalances in brain tissue homeostasis: by affecting synaptic plasticity and the overall neuronal function [1] and/or by triggering exacerbated inflammation, which is a common feature of both chronic and acute CNS disorders [29, 30].

Known for its toxicity, carbon monoxide (CO) is a gaseous molecule, endogenously produced by the degradation of haem catalysed by haem oxygenase (HO) [31]. This reaction yields $\mathrm{CO}$, along with free iron $\left(\mathrm{Fe}^{2+}\right)$ and biliverdin [31, 32]. $\mathrm{HO}$ has two described isoforms: the constitutive form HO-2 and HO-1, an inducible isozyme, whose expression is activated by a wide array of stress signals [32]. $\mathrm{CO}$ is a signalling molecule, and the $\mathrm{CO} / \mathrm{HO}-1$ axis is protective in several biological contexts, modulating inflammation, apoptosis, cell differentiation and metabolism [33-35] CO-derived ROS production primes the anti-inflammatory player peroxisome proliferator-activated receptor gamma co-activator 1 $\alpha$ (PPAR- $\gamma$ ) in macrophages [36] and lung [37], decreasing downstream pro-inflammatory mediators. $\mathrm{CO}$ also regulates the activities of mitogen-activated protein kinase (MAPK), resulting in lower secretion of key cytokines, which limits pro-inflammatory stimulation [38-40]. In CNS, CO has been described as neuroprotective by preventing neuronal cell death in vitro $[41,42]$ and in ischaemia and reperfusion in vivo models $[43,44]$. In glial cells, $\mathrm{CO}$ is cytoprotective in astrocytes, namely $\mathrm{CO}$ inhibited apoptosis by regulating mitochondrial membrane permeabilization [45], by improving oxidative metabolism [46] and by modulating P2X7 receptors [47]. Likewise, vasodilation is promoted by $\mathrm{CO}$ via astrocytic glutamate receptors [48]. In microglia, CO reduces inflammatory response induced by LPS (lipopolysaccharide), thrombin or interferon $\gamma($ IFN- $\gamma$ ) $[38,49]$. This anti-inflammatory effect occurs by chemically inhibiting PI3K (phosphoinositide 3-kinase) an ERK (extracellular-signal-regulated kinase) [38, 49]. CO also decreases neuroinflammation in microglia via modulation of cell metabolism, namely by enhancing mitochondrial respiration [50]. In vivo models of CNS disorders have highlighted the potential of $\mathrm{CO}$ as a regulator of local brain immunity. In fact, $\mathrm{CO}$ treatment ameliorates tissue inflammation and limits cell loss and 
disease progression in models for autoimmune encephalomyelitis [51] and haemorrhagic stroke [52].

Herein, we aimed to assess how $\mathrm{CO}$ alters microglia function and paracrine communication with the surrounding cellular milieu, particularly with neurons. In fact, CO's role on microglia-neuron direct communication has not been an object of study to the best of our knowledge. Thus, the present work targets the underlying molecular mechanisms of this intercellular communication primarily by the analysis of neuroinflammatory and neurotrophic factors in microglial secretome. Then, it is explored how secretome from CO-treated microglia affects neuronal survival, morphology and function.

The therapeutic advent for $\mathrm{CO}$ is supported by the increasing number of applications [32] and can be clinically implemented by the development of CO-releasing molecules (CORMs) to circumvent several limitations regarding CO's pharmacological delivery, namely safety and tissue specificity [53-55]. Herein, a novel CORM, ALF-826, is used to assess the impact of $\mathrm{CO}$ on microglial neuroinflammation and neurotrophism, and on microglia-neuron communication. ALF-826 is a molybdenum carbonyl complex which spontaneously releases $\mathrm{CO}$ in biological media, and it has been recently demonstrated that ALF-826 is anti-inflammatory in BV2 microglial cells when applied after LPS challenge [56]. Moreover, molybdenum is a trace element that is essential for the function of at least three human enzymes: xanthine oxidase, sulphite oxidase and aldehyde oxidase.

\section{Methods}

\section{Materials}

All cell culture plastics were purchased from Corning (Corning, NY) and Sarstedt (Sarstedt, Germany). Chemicals of analytical grade were obtained from Sigma-Aldrich (St Louis, MI), unless stated otherwise. All used antibodies and their respective technique are described in Supplementary Table 1.

\section{Cell Line Cultures}

BV2 murine microglia cell line was kindly supplied by Dr Ana Raquel Santiago (Faculdade de Medicina da Universidade de Coimbra). All experiments with BV2 cells were performed up to the passage nos. 33-37. Cells were grown in RPMI-1640 medium (Sigma-Aldrich), supplemented with $10 \%$ foetal bovine serum (FBS, Thermo-Fisher Scientific), $4 \mathrm{mM}$ L-glutamine (Thermo-Fisher Scientific), penicillin $(100$ units $/ \mathrm{mL})$ and streptomycin $(100 \mu \mathrm{g} / \mathrm{mL})$ (Thermo-Fisher Scientific). CAD mouse catecholaminergic neuronal cell line was gently provided by Dr Federico Herrera (Faculdade de Ciências da Universidade de Lisboa). All experiments with CAD cells were performed up to the passage nos. 25-30. DMEM/F12 was used as basal medium (Thermo-Fisher Scientific) and supplemented with 8\% FBS (Thermo-Fisher Scientific), penicillin (200 units $/ \mathrm{mL}$ ) and streptomycin $(200 \mu \mathrm{g} / \mathrm{mL})$ (Thermo-Fisher Scientific). For differentiation, growing medium was replaced by a serumfree DMEM/F12 supplemented solution. SH-SY5Y human neuroblastoma was used. For cell maintenance, DMEM/ F12 was used as basal medium (Thermo-Fisher Scientific) and supplemented with 8\% FBS (Thermo-Fisher Scientific), penicillin $(200$ units $/ \mathrm{mL})$ and streptomycin $(200 \mu \mathrm{g} / \mathrm{mL})$ (Thermo-Fisher Scientific). All cell lines were maintained in a humified incubator at $37{ }^{\circ} \mathrm{C}$ and $5 \% \mathrm{CO}_{2}$.

\section{Primary Cell Cultures}

Animals were housed in standard laboratory conditions with free access to water and standard rodent chow. All animal experiments were performed with the approval of the i3S Animal Ethics Committee and in accordance with European Union Directive on the protection of animals used for scientific purposes (2010/63/EU) and the Portuguese Law that transposes it (Decreto-Lei n. ${ }^{\circ} 113 / 2013$ ). All efforts were made to minimise animal suffering. Neuron hippocampal cell cultures were isolated from embryonic day $18 \mathrm{Wistar}$ rats. Pregnant females were euthanized in a $\mathrm{CO}_{2}$ chamber; their abdominal region was pulverised with $70 \%$ ethanol and opened using surgical scissor and tweezers. Embryos were retrieved, decapitated, and the brains were placed in a Petri dish containing Hank's Balanced Salt Solution (HBSS, Thermo-Fisher Scientific) supplemented with $100 \mu \mathrm{g} / \mathrm{mL}$ gentamicin (Lonza). The brains were dissected under a magnifying glass, and the hippocampi were collected. Hippocampal tissue was incubated for $15 \mathrm{~min}$ at $37^{\circ} \mathrm{C}$ with $1.5 \mathrm{mg} / \mathrm{mL}$ of trypsin (Thermo-Fisher Scientific), and after allowing the hippocampi to sediment, the supernatant was removed and a 10\% FBS (Thermo-Fisher Scientific) solution was added. Following gentle agitation, the supernatant was discarded, and a washing step was performed with fresh HBSS. The supernatant was again removed, and neurobasal medium (Thermo-Fisher Scientific) supplemented with $25 \mu \mathrm{M}$ glutamate (Sigma-Aldrich), $0.5 \mathrm{mM}$ L-glutamine (Thermo-Fisher Scientific), $100 \mu \mathrm{g} / \mathrm{mL}$ gentamicin (Lonza) and 1\% B27 supplement (Thermo-Fisher Scientific) was added, and the tissue was dissociated by thorough pipetting. The suspension was then put through a $70-\mu \mathrm{m}$ cell strainer and collected, and cells were counted with a Neubauer counting chamber. Neurons were plated on 24 multi-well plates $(100,000$ cells/well), on top of poly-D-lysine (PDL, $1 \mathrm{mg} / \mathrm{mL}$, Sigma-Aldrich) coated glass coverslips. Cells grew in vitro for 10 days before any analysis was carried forward, with fresh medium being added after7 days in culture.

Mixed glial cell (MGC) cultures were obtained from cortices of 2-day old Wistar pups. Animals were quickly 
sacrificed, with brains being removed and dissected consequently in HBSS (Thermo-Fisher Scientific) with penicillin $(100$ units $/ \mathrm{mL})$ and streptomycin $(100 \mu \mathrm{g} / \mathrm{mL})$ (Thermo-Fisher Scientific). White matter and meninges were discarded, and the remaining tissue was collected and mechanically homogenised. The cell suspension was passed through a 25-gauge syringe and incubated with $0.1 \mathrm{U} / \mathrm{mL}$ DNAse I (Zymo) and $1.5 \mathrm{mg} / \mathrm{mL}$ trypsin (Thermo-Fisher Scientific) for $15 \mathrm{~min}$ at $37^{\circ} \mathrm{C}$. After incubating, trypsin was inactivated by adding DMEM GlutaMAX (ThermoFisher Scientific) supplemented with penicillin (100 units/ $\mathrm{mL})$, streptomycin $(100 \mu \mathrm{g} / \mathrm{mL})$ (Thermo-Fisher Scientific) and 10\% FBS (Thermo-Fisher Scientific), and the suspension was centrifuged afterwards $(550 \mathrm{~g}, 15 \mathrm{~min})$. The resulting supernatant was carefully discarded, and the pellet was resuspended in the same medium and put through a 100$\mu \mathrm{m}$ cell strainer. The obtained suspension was distributed equally into $75-\mathrm{cm}^{2} \mathrm{t}$-flasks ( 2 brain per flask) to make a total media of $10 \mathrm{~mL}$ per flask. Prior to adding the cells, the t-flasks were coated with $1 \mathrm{mg} / \mathrm{mL}$ PDL (Sigma-Aldrich) for $1 \mathrm{~h}$. The cell medium was changed every other day.

Primary microglia cells were obtained from 10-day-old MGC cultures, which were placed on an orbital shaker incubator for $2 \mathrm{~h}$ at $200 \mathrm{rpm}$. The cell suspension was collected and centrifuged ( $260 \mathrm{rcf}, 10 \mathrm{~min}$ ), and the supernatant was discarded. The pellet was resuspended in DMEM/F12 (Thermo-Fisher Scientific), and cells were counted using a Neubauer counting chamber. Microglia cells were always obtained from either 10 (shake 1) or 17 days in vitro (DIV, shake 2) MGC cultures. Older cell cultures were not considered for this. Microglial cells were plated on 24 multi-well plates, with DMEM/F-12 GlutaMAX (Thermo-Fisher Scientific) and 4\% L-glutamine (Sigma-Aldrich), penicillin (10 units $/ \mathrm{mL})$ and streptomycin $(10 \mu \mathrm{g} / \mathrm{mL})$ (Thermo-Fisher Scientific) and 10\% FBS (Thermo-Fisher Scientific) supplement.

\section{Cell Culture Treatments}

For neuroinflammatory assay, BV2 cells were seeded onto multi-well plates $\left(10 \times 10^{4}\right.$ cells per well), treated with ALF$826(50 \mu \mathrm{M})$ for $24 \mathrm{~h}$ before LPS (lipopolysaccharide, $500 \mathrm{ng}$ / $\mathrm{mL}$, Sigma-Aldrich) was added for another $24 \mathrm{~h}$. Subsequently, the BV2 microglia conditioned media was collected and centrifuged for $5 \mathrm{~min}, 500 \mathrm{~g}$ (Fig. 1A). Simultaneously, CAD neurons were seeded $\left(6 \times 10^{4}\right.$ cells per well $)$, and $24 \mathrm{~h}$ later, differentiating medium was added. After $48 \mathrm{~h}$ of differentiation, neuronal supernatant was removed, and CAD were challenged for 1 day with BV2 microglia-conditioned media.

For neurotrophic assay, BV2 cells $\left(10 \times 10^{4}\right.$ cells per well $)$ were treated for $48 \mathrm{~h}$ with ALF-826 $(50 \mu \mathrm{M})$, then medium was collected and centrifuged ( $5 \mathrm{~min}, 500 \mathrm{~g}$ ). The conditioned medium (microglial culture supernatant) was added into 48 h-differentiated CAD neuronal culture. $t$-BHP at 7.5 and $10 \mu \mathrm{M}$ was added into the $\mathrm{CAD}$ neuronal culture to promote cell death for $24 \mathrm{~h}$, with or without the presence of BV2 microglia-conditioned medium, when mentioned (Fig. 7A). To assess the effect of purinergic signalling on neuron-microglia communication, $48 \mathrm{~h}$-differentiated CAD neurons were treated, simultaneously, with microglia-conditioned media and adenosine receptor antagonists SCH-58261 (1 and $5 \mu \mathrm{M})$ and Dipropylcyclopentylxanthine (DPCPX, 25 and $50 \mu \mathrm{M}$ ). Primary culture of microglia derived from 10 or 17 DIV MGC cultures was collected and plated on 24 multi-wells $\left(7 \times 10^{5}\right.$ cells/well). Twenty-four hours after seeding, cells were treated with $50 \mu \mathrm{M}$ of ALF-826 for $24 \mathrm{~h}$ then with $10 \mathrm{ng} / \mathrm{mL}$ of LPS for another $24 \mathrm{~h}$. Microglia medium was collected and centrifuged at $500 \mathrm{~g}$ for $5 \mathrm{~min}$, and its supernatant was used as conditioned medium. Fifty percent volume of the neuronal medium of 10 DIV hippocampal neurons cultured in 24 multiwell plates was replaced by microglia-conditioned media. Neuronal cells were analysed following $24 \mathrm{~h}$.

\section{Reagents and Solution Preparation}

ALF-826 was provided by Proterris (Portugal) Lda. Stock solutions of $2.5 \mathrm{mM}$ were prepared by dissolving the compound in Dimethyl sulfoxide (DMSO, Sigma-Aldrich) and diluting it $1 / 10$ in a $\mathrm{NaHCO}_{3}$ solution $(0.1 \mathrm{mM}, \mathrm{pH} 8.3)$. ALF-826 was then filtered, aliquoted and stored at $-80{ }^{\circ} \mathrm{C}$ at a final concentration of $2.5 \mathrm{mM}$. ALF- 826 has a half-life of $37 \mathrm{~min}$ in Hepes 7.4 buffer. Its cytotoxicity is negligible at $100 \mu \mathrm{M}$ (98\% survival of RAW246.7 macrophages at $24 \mathrm{~h}$ ), and its $\mathrm{CO}$ effective load is ca. 3 equivalents of $\mathrm{CO}$ per mol (information provided by Proterris (Portugal) Lda.).

For the preparation of the depleted form of ALF-826 (iALF-826), the compound was dissolved in DMSO, left for $24 \mathrm{~h}$ at room temperature to allow for the dissociation of the $\mathrm{CO}$ groups from the molecular scaffold and subsequently diluted in $1 / 10$ in a $\mathrm{NaHCO}_{3}$ solution $(0.1 \mathrm{mM}, \mathrm{pH} 8.3)$. iALF-826 was then filtered, aliquoted and stored at $-80^{\circ} \mathrm{C}$ at a final concentration of $2.5 \mathrm{mM}$.

Fresh $\mathrm{CO}$ gas solutions of $\mathrm{CO}$ were prepared by saturating PBS, bubbling $100 \%$ of CO compressed gas (Linde, Germany) for $30 \mathrm{~min}$, yielding a $10^{-3} \mathrm{M}$ stock solution. $\mathrm{CO}$ concentration in solution was measured spectrophotometrically, by the conversion of deoxymyoglobin to carbon monoxymyoglobin, in an assay previously described by Motterlini et al. [53].

\section{Flow Cytometry}

\section{Neuronal Cell Viability Assay}

CAD neurons were collected by trypsinisation and stained with $1 \mathrm{ng} / \mathrm{mL}$ of propidium iodide (PI, Thermo-Fisher Scientific) for $15 \mathrm{~min}$ at $37^{\circ} \mathrm{C}$. Cell viability was analysed by 
flow cytometry using the FACS Canto II (BD Biosciences). A 488-nm laser line was used for excitation, and PI was read in the FL-3 channel in the linear scale. Appropriate controls, such as positive staining controls and unstained samples, were always carried out. Data analysis was performed with the FlowJo software (BD Biosciences).

\section{Microglia Reactivity Assay}

BV2 cells were collected by scrapping and stained with $500 \mathrm{ng} / \mathrm{mL}$ of PE/Cy7 (Phycoerythrin/Cyanine 7) antimouse $\mathrm{CD} 11 \mathrm{~b}$ antibody (BioLegend) for $15 \mathrm{~min}$ at $37^{\circ} \mathrm{C}$. Microglia reactivity was analysed by flow cytometry using FACS Canto II (BD Biosciences). A 488-nm laser line was used for excitation, and reactivity levels were determined by reading sample's PE/Cy7 Median Fluorescence Intensity in the FL-4 channel in the linear scale. Appropriate controls, such as positive staining controls and unstained samples, were always carried out. Subsequent data analysis was performed using FlowJo software (BD Biosciences).

\section{Enzyme-Linked Immunosorbent Assay}

Enzyme-linked immunosorbent assay (ELISA) was performed to measure tumour necrosis factor $\alpha(\mathrm{TNF}-\alpha)$, interleukin-1 $\beta$ (IL-1 $\beta$ ) and IL-10 microglia supernatant levels, using the respective Standard ABTS ELISA Development Kits (PeproTech). For glial cell-derived neurotrophic factor (GDNF) and brain-derived neurotrophic factor (BDNF), different kits were used: (GDNF ELISA Kit (Abcam) and Human/Mouse BDNF DuoSet ELISA (R\&D)). Microglia culture media was collected and centrifuged to remove cellular debris, and the pellet was discarded. The resulting supernatant was kept at $-80{ }^{\circ} \mathrm{C}$ until analysis. All experiments were performed in accordance with the respective manufacturer's instructions. Absorbance values were measured at $415 \mathrm{~nm}$, with wavelength correction set at $560 \mathrm{~nm}$, using an Infinite F200 PRO microplate reader (Tecan).

\section{Griess Reaction Assay}

Nitrite of microglia supernatant was quantified by Griess reaction colorimetric test. Microglia culture media was collected and centrifuged to remove cellular debris and subsequently incubated with Griess reagent (1:1 ratio, SigmaAldrich) for $10 \mathrm{~min}$ at room temperature (RT), protected from light. Absorbance was measured at $540 \mathrm{~nm}$ using an Infinite F200 PRO microplate reader (Tecan). Nitrite concentration was calculated with reference to a standard curve generated with known concentrations of sodium nitrite ( $\mathrm{NaNO}_{2}$, Sigma-Aldrich).

\section{Immunoblotting}

Cell extracts were washed several times with ice-cold PBS and lysed with RIPA (Radioimmunoprecipitation assay) buffer (50 mM Tris-HCl, pH 6.8, $50 \mathrm{mM} \mathrm{NaCl}$ (w/v), $0.1 \%$ SDS (w/v), $1 \%$ sodium deoxycholate (w/v), $1 \%$ Triton X-100 $(\mathrm{v} / \mathrm{v}), 10 \%$ glycerol $(\mathrm{v} / \mathrm{v})$ and $1 \%$ protease inhibitor cocktail $(\mathrm{v} / \mathrm{v})$. Protein concentration was determined with a Pierce BCA Protein Assay Kit (Thermo-Fisher Scientific), following manufacturer's instructions. Absorbance values were registered at $560 \mathrm{~nm}$ using an Infinite F200 PRO microplate reader (Tecan). A bovine serum albumin (BSA, Merck) standard curve was constructed to determine protein concentration.

Equal amounts of protein were separated by sodium dodecyl sulphate-polyacrylamide gel electrophoresis (SDSPAGE) on a polyacrylamide gel (10\% gel unless stated otherwise), with a NZYColour Protein Marker II (NZYtech) being used as band size reference. Proteins were then electrically transferred onto an Amersham Protran $0.45 \mathrm{NC}$ nitrocellulose membrane (GE LifeSciences) and blocked with $5 \%(\mathrm{~m} / \mathrm{v})$ BSA in T-TBS for $1 \mathrm{~h}$. Membranes were labelled consecutively with primary and secondary antibodies as indicated in Supplementary Table 1. Immunoblots were exposed to ECL Clarity Western Detection Reagent (BioRad) $5 \mathrm{~min}$, and the reactive bands were detected after the membranes were exposed to X-ray film (Chemidoc Touch Imaging System, Bio-Rad). The resulting area and intensity of the bands were quantified with ImageLab software (Bio$\mathrm{Rad}) . \beta$-actin was used as internal loading control unless otherwise is stated.

\section{ROS Generation Assay}

ROS, in particular hydrogen peroxide generation, were measured following the conversion of $2^{\prime}, 7^{\prime}$-dichlorofluorescein diacetate (H2DCFDA) (Invitrogen) to fluorescent $2^{\prime}, 7^{\prime}$-dichlorofluorescein (DCF). BV2 microglia cells were seeded $\left(9 \times 10^{3}\right.$ cells/well) on 96 -well black flat bottom plates and treated with ALF-826 and/or LPS, as described previously. After treatment, microglia supernatant was removed, and cells were washed twice with ice-cold PBS and treated for 15 min with $5 \mu \mathrm{M}$ of H2DCFDA. Cell extracts were subsequently washed, and fluorescence intensity was measured using a Tecan Infinite F200 PRO microplate reader $\left(\lambda_{\mathrm{ex}} 485 \mathrm{~nm} / \lambda_{\mathrm{em}} 530 \mathrm{~nm}\right)$. ROS generation was calculated as an increase over baseline levels, determined for untreated cells and normalised to total protein quantification for each condition.

\section{Immunofluorescence Microscopy}

Cells growing on glass coverslips were fixated with $4 \%(\mathrm{v} / \mathrm{v})$ PFA (paraformaldehyde) and 4\% (w/v) 


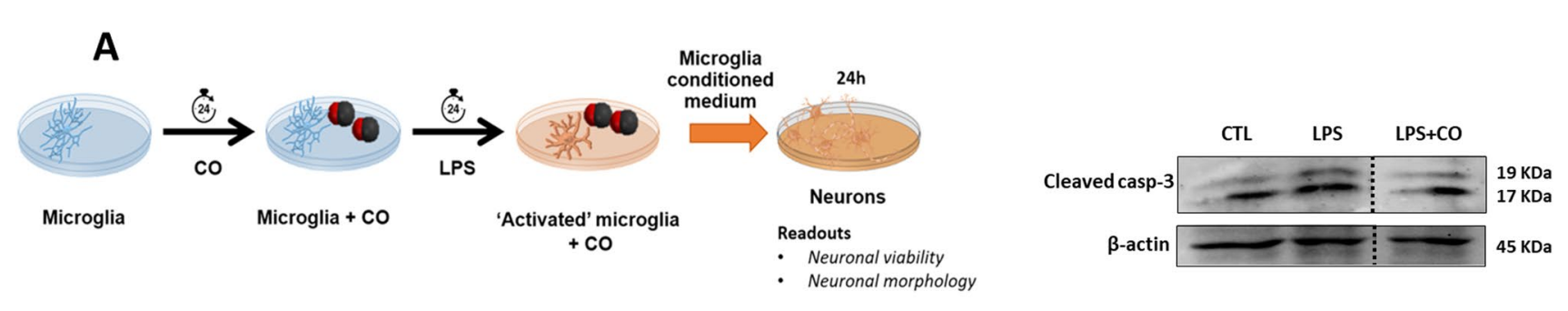

B

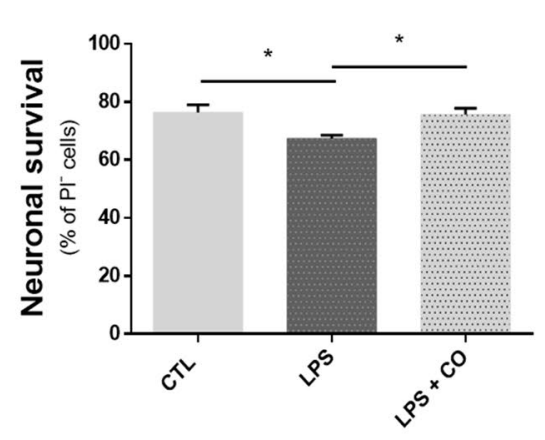

D
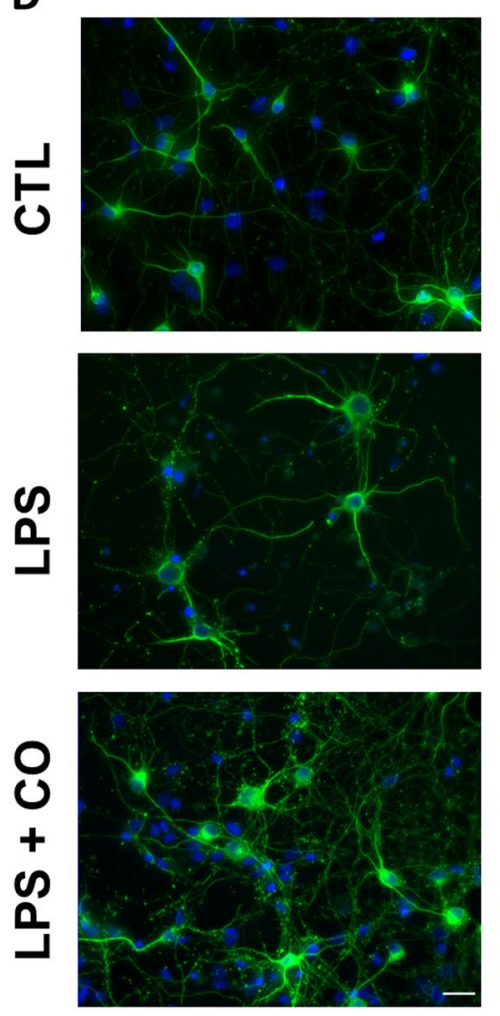
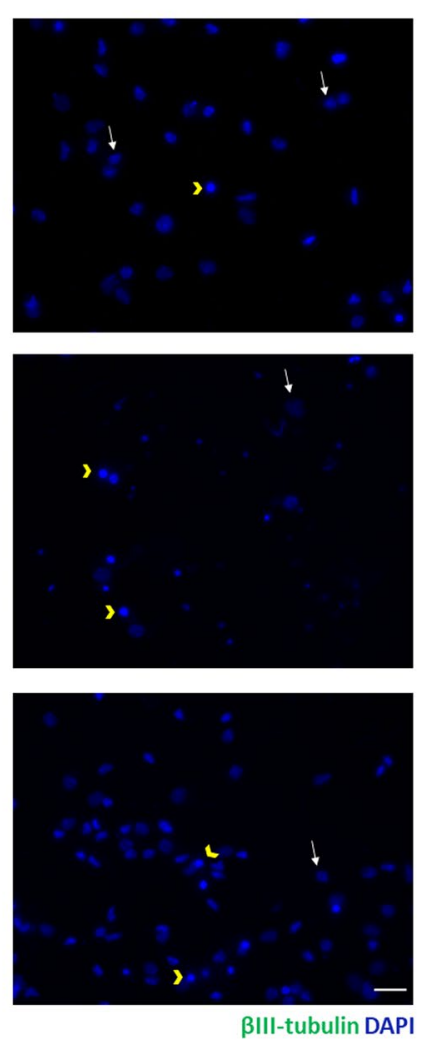

C

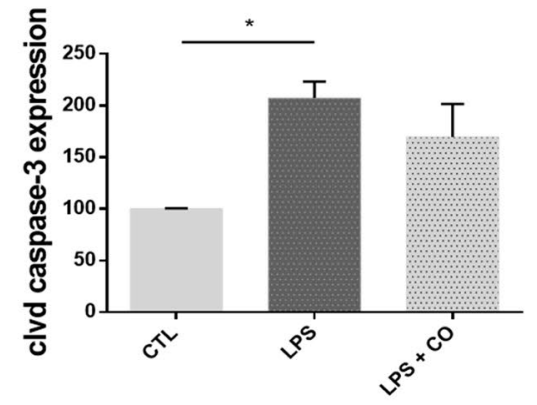

E

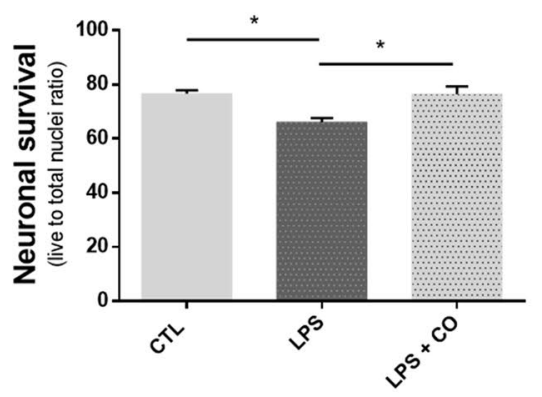

sucrose solution ( 20 min at RT), permeabilized with $0.3 \%(\mathrm{v} / \mathrm{v})$ Triton X-100 solution (15 min, RT) and then blocked with BSA $1 \%(\mathrm{w} / \mathrm{v})$ and Triton X-100 $0.1 \%(\mathrm{w} / \mathrm{v})$ for half hour at RT. Later, cells were probed with primary (2-h incubation) and secondary $(1 \mathrm{~h})$ antibodies, as described in Supplementary Table 1. Coverslips were mounted onto glass slides with Prolong mounting medium (with DAPI 1:1000 (4',6-diamidino-2-phenylindole, Thermo-Fisher Scientific). Washing with ice-cold PBS was always performed between steps. All antibodies used were diluted in $1 \%(\mathrm{v} / \mathrm{v})$ BSA and $0.1 \%(\mathrm{v} / \mathrm{v})$ Triton X-100 solution. Fixating, permeabilizing and blocking solutions were prepared in PBS. Images were captured with a Zeiss $\mathrm{Z} 2$ microscope, with at least five random micrographs being acquired, unless otherwise stated. 
4Fig. 1 Carbon monoxide (CO) treatment in microglia attenuates neuronal cell death induced by conditioned medium from LPS-activated microglia. BV2 microglia were treated with ALF-826 $(50 \mu \mathrm{M})$ for $24 \mathrm{~h}$ and with LPS $(500 \mathrm{ng} / \mathrm{mL})$ for another $24 \mathrm{~h}$. 'Activated' microglia are cells treated with LPS. CAD neuronal culture was subsequently incubated with microglia-conditioned medium (A). Cell viability was assessed by propidium iodide staining quantification in flow cytometry (B) or by cleaved caspase 3 protein expression by western blotting (C). For $1 \mathbf{B}, n=6-7$, error bars represent mean \pm SEM. One-way ANOVA test with Tukey's multiple comparison test was performed ( $p$ value $=0.0163, F=5.38$ ). The densitometry of cleaved caspase-3 (average of fragments 17 and $19 \mathrm{kDa}$ densitometry) expression was normalised for $\beta$-actin and is presented as percentage relative to the control. Representative membranes for the experiments are displayed (C). $n=4$, error bars represent mean \pm SEM. Kruskal-Wallis test with Dunn's multiple comparison was performed ( $p$ value $=0.0214$, Kruskal-Wallis statistic $=6.006$ ) . $\mathrm{CO}$ provides neuroprotection in primary hippocampal neurons via modulation of primary microglia. Rat hippocampal neurons were challenged with conditioned medium from primary microglia cells for $24 \mathrm{~h}$ and immunostained with $\beta$ III-tubulin primary antibody (green) and DAPI (nuclei in blue), scale bar $=25 \mu \mathrm{m}(\mathbf{D})$. Cell viability was assessed by counting total and apoptotic neurons. White arrows highlight the nucleus of live neurons, and yellow arrowheads highlight the nucleus of dead neurons. The live/total nuclei ratio was registered and is presented in $\mathbf{E}$. $n=9$; error bars represent mean \pm SEM. One-way ANOVA test with Tukey's multiple comparison test was performed $(p$ value $=0.0011, F=9.229)$

\section{Neuronal Morphology Analysis}

Neurite tracing was done using the NeuronJ Fiji plug-in (Erik Meijering) and following instructions present in an online manual provided by the developer (https://image science.org/meijering/software/neuronj/manual/). Neurites were labelled and clustered as primary (originating from cell soma), secondary (from a primary neurite) and tertiary (from a secondary neurite) processes. Software calculation determined total number of neurites per field as well as individual neurite length. Similarly, neuron skeleton reconstruction and Sholl analysis (quantification of intersections at concentric spheres originating from cell soma to $150 \mu \mathrm{m}$, with radius every $5 \mu \mathrm{m}$ ) were performed using the Simple Neurite Tracer Fiji plug-in, in accordance to instructions present in online tutorials (https://imagej.net/Simple_Neurite_ Tracer). For each individual experiment, four to six images were analysed for each glass slide.

\section{High-Performance Liquid Chromatography}

To quantify microglial adenosine, supernatant from BV2 cells was collected from each well, deproteinized in PCA $3 \mathrm{M}$ and centrifuged at $4{ }^{\circ} \mathrm{C}, 10 \mathrm{~min}$ and $13,000 \mathrm{~g}$. Then the supernatants neutralised (pH 6.8-7.2) with $\mathrm{KOH} /$ Tris (Sigma-Aldrich) as previously described by Conde and Monteiro (2004). Adenosine was quantified by HPLC with UV detection at $254 \mathrm{~nm}$. The HPLC system consisted of an LC 10-AD pump, SIL-20AC autosampler, SPD-20 A/
AV UV-VIS wavelength detector and Class VP software to analyse the chromatograms (Shimadzu, Kyoto, Japan). The analytical column was a Lichrospher 100 RP-18 (125 $4 \mathrm{~mm}$, i.d., particle size $5 \mu \mathrm{m}$, Merck, Madrid, Spain) protected by LichroCART 4-4 guard-columns (Merck, Madrid, Spain). The mobile phase was $100 \mathrm{~mm} \mathrm{KH2PO} 4$ with 15\% methanol, pH 6.5, run at a flux of $1.75 \mathrm{ml} \mathrm{min}{ }^{-1}$. External standards were prepared under the same conditions as the biological samples and adenosine identification, and quantification was made against the standards. Adenosine supernatant levels were normalised to total protein microglial content.

\section{Statistical Analysis}

Results are presented as mean \pm standard error of the mean (SEM), with at least three biological replicates being performed for all experiments. All statistical analyses were performed using the Prism 6.0 software (GraphPad). Kolmogorov-Smirnov test was used to check data normality, and subsequent parametric or non-parametric tests were used to assess data statistical significance.

\section{Results}

\section{Conditioned Medium from CO-Treated Microglia Provides Neuroprotection to Neuronal Culture Against Cell Death}

In order to assess the role of carbon monoxide (CO) on remote neuron-microglia communication, a conditioned medium approach was used, which allows to evaluate the impact of microglia secretome on neuronal function and survival. BV2 microglia were pre-treated with $50 \mu \mathrm{M}$ ALF826 for $24 \mathrm{~h}$ and then incubated or not with $500 \mathrm{ng} / \mathrm{mL}$ of LPS (lipopolysaccharide), a TLR-4 (toll-like receptor) agonist, classically used to elicit an inflammatory-like response. After 1 day, microglia medium was collected and added into the catecholaminergic neuronal cell line CAD culture for 24 h, challenging cell survival (Fig. 1A). Neuronal cell survival assessment was based on membrane integrity (propidium iodide internalisation) by flow cytometry (Fig. 1B). Viability of CAD neuronal cells exposed to conditioned media derived from inflammatory microglia decreased, and this effect was partially prevented whenever BV2 microglia were pre-treated with ALF-826 (Fig. 1B). In addition, expression of neuronal cleaved caspase-3 (Fig. 1C), which is a classical biomarker of apoptosis, was quantified by western blot. Accordingly, supernatant derived from CO-treated microglia also tends to decrease the levels of cleaved caspase- 3 in neurons. These data indicated that $\mathrm{CO}$ decreases microglia release of neurotoxic factors, limiting neuronal cell death. 
The data obtained in BV2 microglia-CAD neuron conditioned media protocol were then validated in a more physiologically relevant model: hippocampal neuronal and microglia rat primary cultures. Primary cultures of microglia were treated with ALF-826 $(50 \mu \mathrm{M}, 24 \mathrm{~h})$ and LPS $(10 \mathrm{ng} / \mathrm{mL}$, $24 \mathrm{~h}$ ), and the supernatant was collected, centrifuged and subsequently incubated 1:1 (volume) with neuronal media to challenge 10 days in vitro (DIV) hippocampal neurons. To assess neuronal cell death in primary cultures, neurons were immunolabelled with an antibody against $\beta$ III-tubulin (green), the nuclei were stained with DAPI (blue) and cells were imaged using a fluorescence microscope (Fig. 1D). Apoptosis was quantified by counting chromatin-condensed nuclei (Fig. 1E). In accordance with data from Fig. 1B, neurons cultured with inflammatory conditioned media presented a significant decrease in cell survival, which was prevented whenever microglia were pre-treated with ALF-826.

\section{Conditioned Medium from CO-Treated Microglia Improves Neuronal Morphology}

Neuronal morphology of differentiated CAD cell line was analysed for indirect evaluation of neuronal function (Fig. 2). Neurons exposed to inflammatory microglia media presented a decrease in cellular complexity parameters, such as average neurite length (Fig. 2A) and number of neurites per cell (Fig. 2B). Additionally, data from Sholl analysis, which is a quantitative method used for the analysis of neuronal arborization, further indicated the existence of a decrease of the overall neuronal complexity whenever neurons were challenged with inflammatory conditioned media (Fig. 2C, D). Conditioned medium derived from CO-treated microglia prevented the decrease of neurite length and the number of neurites per cell and maintained neurite arborization at similar levels as the non-treated control neurons. Thus, microglial ALF-826 treatment has a paracrine neuroprotective effect on neuronal cells, potentially achieved through modulation of microglial release of neurotoxic proinflammatory factors.

\section{CO Has an Anti-Inflammatory Role on LPS-Challenged BV2 Microglia}

Since CO appears to provide neuroprotection by regulating neuron-microglia remote communication, thus secretome factors were evaluated (Fig. 3). The extracellular levels of the pro-inflammatory factors nitric oxide (NO), tumour necrosis factor $\alpha(\mathrm{TNF}-\alpha)$ and interleukin-1 $\beta$ (IL-1 $\beta$ ) were quantified in microglial supernatant. Griess colorimetric assay, which allows for the quantification of nitrites, an indirect measure of $\mathrm{NO}$, indicated an increase in nitrites concentration following LPS exposure (Fig. 3A). Thus, ALF-826 treatment partially inhibited LPS-induced nitrite release. The decrease of nitrite secretion was dependent on ALF-826 concentration (Figure S1A). TNF- $\alpha$ was quantified by ELISA; $C O$ also prevented the secretion of TNF- $\alpha$ pro-inflammatory cytokine in LPS BV2 (Fig. 3B).

To confirm that the effect of ALF-826 was due to the $\mathrm{CO}$ release and not of the scaffold of the molecule, nitrite levels were also assessed in BV2 cells following treatment with a CO-saturated PBS solution $(50 \mu \mathrm{M})$. In fact, the saturated solution also reverted NO secretion in LPSstimulated microglia (Figure S1B). To further confirm the effect of the CO-releasing molecule, LPS-treated microglia were also incubated with either ALF-826 or with the CO depleted form (iALF-826). Here, while ALF-826 inhibited secretion of NO (Figure S1C) and TNF- $\alpha$ (Figure S1D), iALF-826 did not produce such effect.

It was next addressed whether $\mathrm{CO}$ anti-neuroinflammatory function is present in primary cultures of microglia. Microglial medium was collected for indirect quantification of NO levels by Griess colorimetric assay and of TNF- $\alpha$ and IL- $1 \beta$ levels by ELISA. For NO and IL- $1 \beta$ secreted factors, LPS triggered a significant increase, which was prevented in the presence of ALF-826 pretreatment (Fig. 3C and E). For TNF- $\alpha$, ALF-826 decreases its secretion, but not in a statistically different manner (Fig. 3D). Contrary to BV2 cell line (data not shown), in microglial primary cultures, LPS elicited elevated levels of IL-1 $\beta$, suggesting a strong NLRP3 (NLR Family Pyrin Domain Containing 3 ) inflammasome reaction. Altogether, these results validate that $\mathrm{CO}$ limits exacerbated inflammation, by reducing the levels of secreted pro-inflammatory factors.

To further confirm the anti-inflammatory effect of ALF826 treatment on BV2 function, we quantified the expression of specific microglial reactivity markers: intracellular ROS production and surface receptor $\mathrm{CD} 11 \mathrm{~b}$. The role of $\mathrm{CO}$ on intracellular ROS production was evaluated by H(2)DCFDA staining that increases its fluorescence in the presence of hydrogen peroxide. Likewise, CO limited LPS apparent increase on microglia-derived oxidative stress (Fig. 4A). Finally, expression of surface receptor CD11b was quantified via flow cytometry. CD11b immunoreactivity appears to increase when LPS was administered to microglia and this was reverted by in ALF-826 pre-treatment (Fig. 4B).

Overall, the results suggest that ALF-826 is a strong pharmacological anti-inflammatory agent due to $\mathrm{CO}$ release. In fact, ALF-826 limits the expression and secretion of several pro-inflammatory agents. Thus, CO-promoted non-cell autonomous neuroprotective effect might result from limiting the inflammatory response of microglial cells.

Medium-Derived from 'Resting' Microglia Limits Apoptosis in CAD Neuronal Cells Exposed to Pro-Oxidant Stimulus and Improves Neuronal Morphological Complexity. 

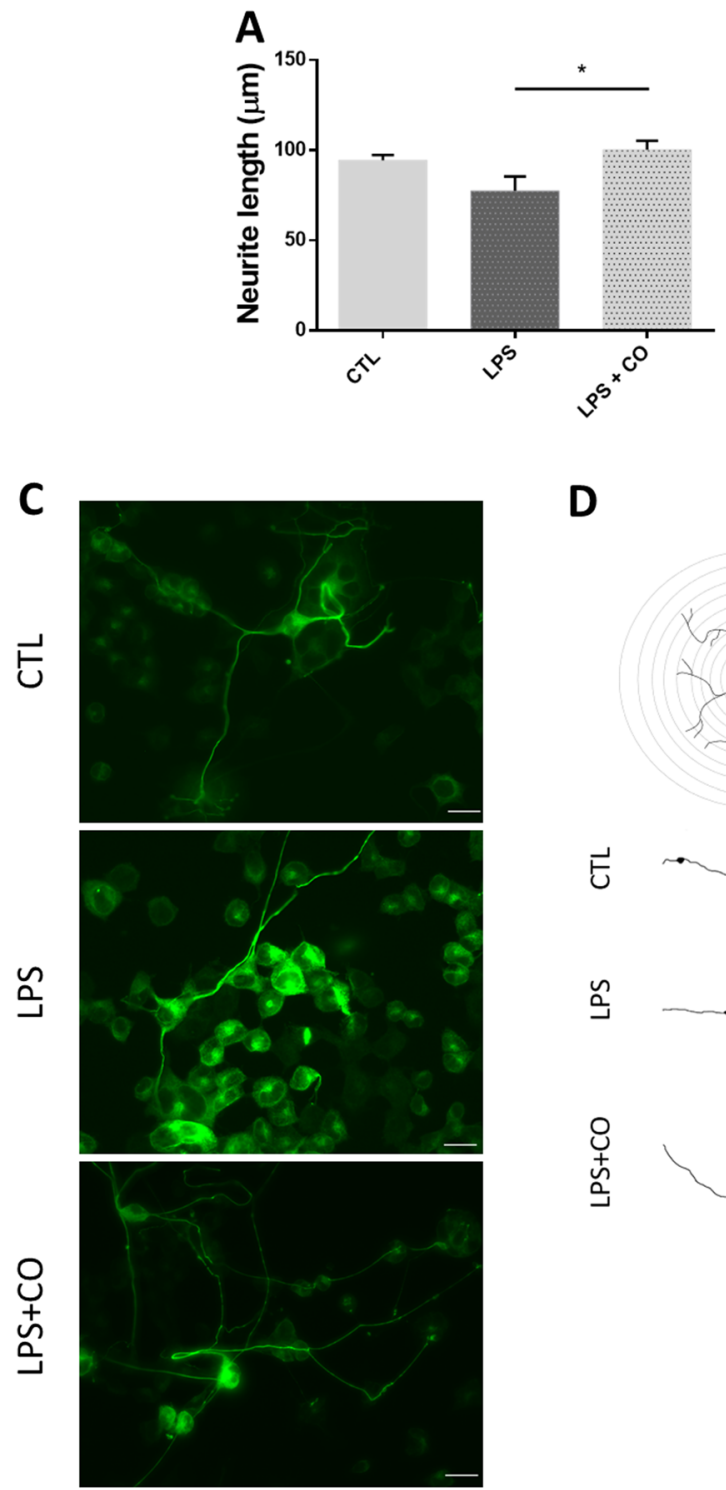

$\beta$ III-tubulin

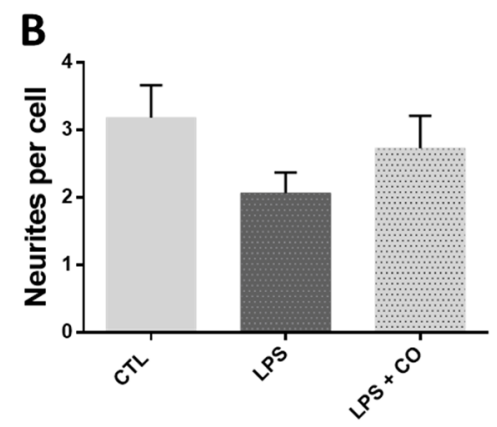

D

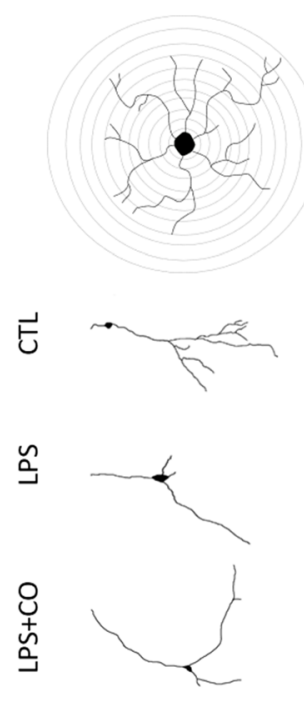

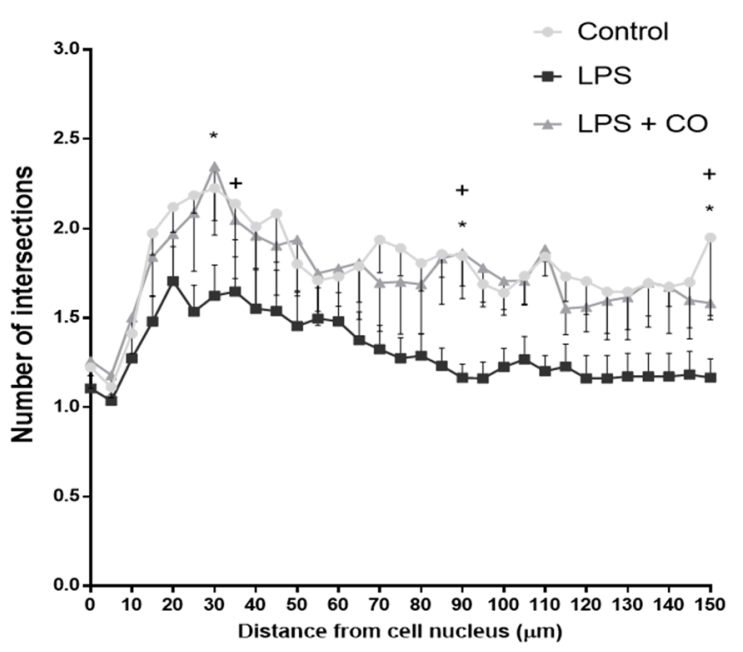

Fig. 2 Administration of CO (ALF-826 at $50 \mu \mathrm{M})$ in microglia limits loss of morphological complexity in neurons exposed to LPSactivated microglia-conditioned medium. CAD neurons treated with BV2 microglia-conditioned media for $24 \mathrm{~h}$ were immunostained with a $\beta$ III-tubulin antibody. Nuclei were stained with DAPI. Following image acquisition, morphological features, such as A neurite length, B number of neurites per cell and D Sholl analysis were assessed from six independent experiments (10-15 cells per condition). C Representative cell pictures for each condition with cells immu-

Other than being the CNS immune guardians, microglia have a crucial role in neuronal basal function, that ranges from modulation of proliferation/differentiation balance during development and trophic support, morphological and synaptic plasticity $[19,57,58]$. Moreover, to the best of our knowledge, there are no data showing CO's role in the modulation of microglia neurotrophic functions. To test the potential microglia-induced neurotrophic effect

nostained with $\beta$ III-tubulin primary antibody (scale bar $=25 \mu \mathrm{m}$ ) and (D) representative tracings of $2 \mathrm{D}$ acquired images were also performed. $n=5-6$; error bars represent mean \pm SEM. For 2 A, one-way ANOVA test with Tukey's multiple comparison test was performed ( $p$ value $=0.0498, F=4.265$ ). For 2B, Kruskal-Wallis test with Dunn's multiple comparison was performed $(p$ value $=0.3532$, Kruskal - Wallis statistic $=2.220$ ). For $2 \mathrm{D}$, two-way ANOVA test with multiple comparisons was performed. *(CTL vs LPS $)$ and ${ }^{+}($LPSCO vs LPS)

and neuroprotection, neuron-microglia-conditioned media approach was used. BV2 microglial cells were treated or not with $50 \mu \mathrm{M}$ ALF- 826 for $48 \mathrm{~h}$, then, microglial supernatant was collected for treating differentiated CAD neurons, which were simultaneously challenged with a pro-oxidant agent (tert-Butyl hydroperoxide, $t$-BHP) to induce neuronal cell death for $24 \mathrm{~h}$ (Fig. 5A). Conditioned medium from microglia and from CO-treated microglia, both reduced levels of 

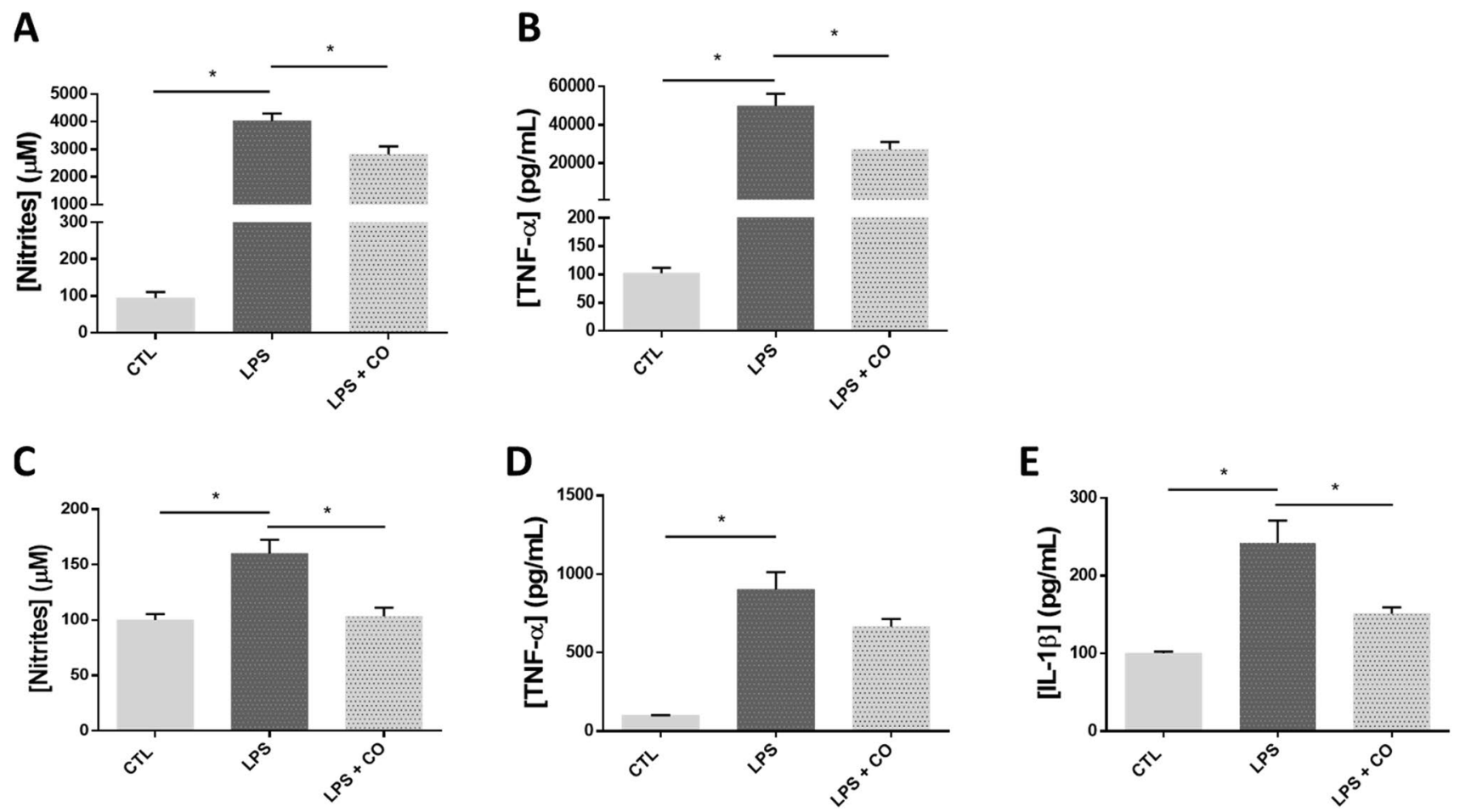

Fig. $3 \mathrm{CO}$ attenuates the inflammatory profile of microglia's secretome. BV2 microglia supernatant was quantified for nitrite levels (A) by Griess colorimetric assay. TNF- $\alpha$ (B) was assessed via ELISA. All results are presented as percentage relative to the negative control. $n=6-8$, error bars represent mean \pm SEM, For $3 \mathbf{A}$ and $\mathbf{B}$, one-way ANOVA test with Tukey's multiple comparison test was performed ( $p$ value $<0.0001, F=75.74$ for $\mathbf{A}),(p$ value $<0.0001, F=32.66$ for $B)$. CO treatment (ALF-826 at $50 \mu \mathrm{M}$ ) inhibits the inflammatory secretome of primary microglial cells. Supernatant from rat primary microglia was collected and secretome was analysed. C Nitrite quan-

neuronal death at 7.5 and $10 \mu \mathrm{M}$ of $t$-BHP (Fig. 5B). These results indicate that microglia-conditioned medium, by itself, has a neuroprotective effect against oxidative stress, while $\mathrm{CO}$ does not further improve microglia-induced neuroprotection.

As microglia have been described to modulate neuronal network dynamics [13], it was next assessed whether CO affects neuronal morphology under basal conditions. In fact, neuronal morphology is a manner to assess neuronal function, and thus an indirectly manner to evaluate neuroprotection. CAD neurons were incubated with conditioned media from control and CO-treated microglia and stained with anti- $\beta$ III-tubulin (green) and with DAPI (nuclei staining) for morphological analysis. Microglial $\mathrm{CO}$ treatment modulates neuronal morphology by increasing the number of neurites per cell (Fig. 6A) and neurite length (Fig. 6B).

Overall, $\mathrm{CO}$ appears to stimulate microglial neurotrophic function by improving neuronal morphology under basal conditions. tification was performed via Griess colorimetric assay, and ELISA assays were utilised for the quantification of TNF- $\alpha$ (D) and IL-1ß (E) levels. All data is presented as percentage relative to the negative control. $n=4-6$; error bars represent mean \pm SEM. For $3 \mathbf{C}$ and $\mathbf{E}$, one-way ANOVA test with Tukey's multiple comparison test was performed $(p$ value $=0.0004, F=13.73$ for $\mathbf{C}),(p$ value $=0.0001$, $F=17.22$ for E) For 3D, Kruskal-Wallis test with Dunn's multiple comparison was performed $(p$ value $=0.0002$, Kruskal-Wallis statistic $=9.846)$

\section{CO Stimulates Microglial Neurotrophic Response}

Lastly, the microglia secretome was analysed to disclose which molecular players were involved in CO-mediated microglia neuroprotection and neuronal morphological modulation. Several candidates described as key players in neurotrophism, and neuronal support were assessed: BDNF (brain-derived neurotrophic factor), GDNF (glial cell-derived neurotrophic factor) and the anti-inflammatory cytokine IL-10. In fact, CO-stimulated microglial IL-10 secretion was demonstrated by ELISA (Fig. 7A). However, $\mathrm{CO}$ administration did not increase BDNF or GDNF secretion (data not shown). This result could point towards a neurotrophic role of $\mathrm{CO}$. In fact, IL-10 overexpression is not only relevant for resolution of inflammation, but also for regulation of cell survival, neurite dynamics, development and synaptogenesis $[59,60]$.

Moreover, our lab has shown in the past that $\mathrm{CO}$ affects astrocyte-to-neuron paracrine communication, promoting neuroprotection via purinergic signalling [61]. Thus, we 


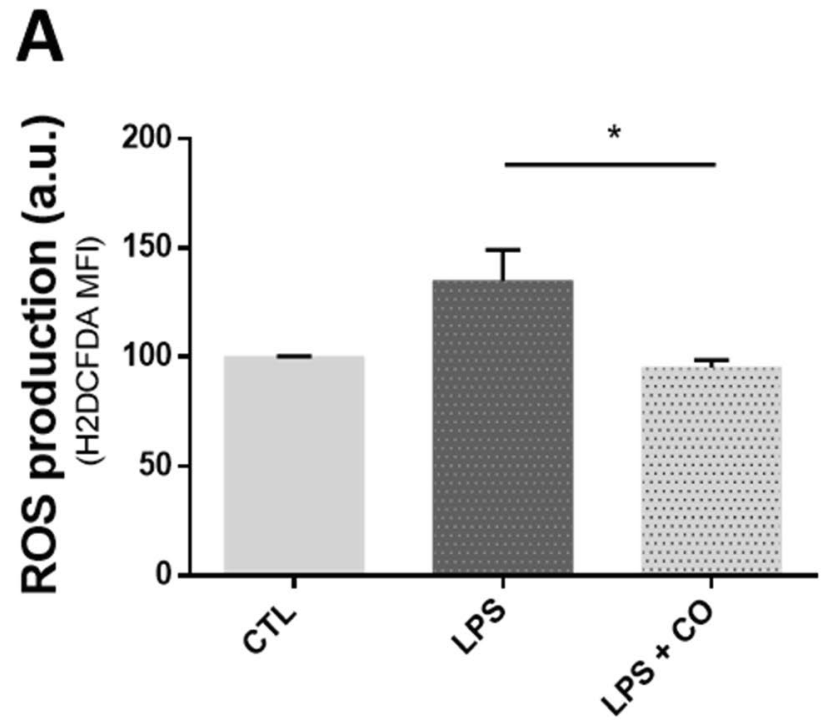

Fig. $4 \mathrm{CO}$ decreases microglial expression of inflammatory reactivity markers. BV2 microglia cells, treated or not with ALF-826 at $50 \mu \mathrm{M}$, were collected and expression of inflammatory markers was assessed. A Intracellular $\mathrm{H}_{2} \mathrm{O}_{2}$ levels were measured by fluorescence using H(2)DCF-DA and normalised to total protein levels. B CD11b surface expression was quantified by $\mathrm{Pe} / \mathrm{Cy} 7$ immunofluorescence

assessed the hypothesis that CO's regulation of microglianeuron remote communication is partially modulated by a similar mechanism (Fig. 7B). High-performance liquid chromatography (HPLC) showed that $\mathrm{CO}$ administration appears to increase microglial adenosine levels in the supernatant (Fig. 7C). Also, the protein levels of surface enzyme CD73, which converts purine mononucleotides into nucleosides, were also increased in CO-treated microglia (Fig. 7D). Hence, $\mathrm{CO}$ may reinforce neuron-microglia communication via purinergic mechanisms.

\section{Chemical Inhibition of Microglia-to-Neuron Purinergic Communication Reverts CO's Neuroprotective Properties}

To validate whether the $\mathrm{CO}$ neuroprotective and morphogenic effect are caused by an increase in levels of adenosine in the microglial supernatant, neurons were treated with $\mathrm{A}_{2 \mathrm{~A}} \mathrm{R}$ antagonist SCH-58261 (1 or $\left.5 \mu \mathrm{M}\right)$ or with $\mathrm{A}_{1} \mathrm{R}$ antagonist 8-Cyclopentyl-1,3-dipropylxanthine (DPCPX) $(25$ or $50 \mu \mathrm{M})$ and simultaneously incubated with microgliaconditioned media (Fig. 5A). Then, neuronal morphometric parameters and neuronal survival were analysed. Inhibition of adenosine receptors did not have any effect on neuronal survival (data not shown). Nevertheless, the increase in neurite length observed whenever CAD cells were treated with conditioned media from CO-treated microglia was reverted whenever an adenosine receptor antagonist was used (Fig. 8B), indicating that the $\mathrm{CO}$ effect is mediated via
B

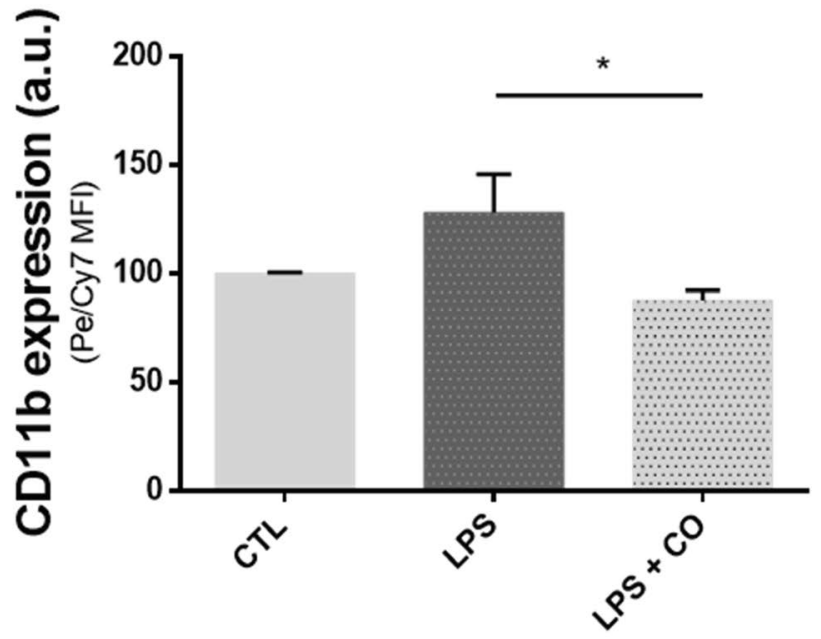

median fluorescence intensity via flow cytometry. Results in $\mathbf{A}$ and $\mathbf{B}$ are presented as percentage relative to the negative control. $n=3-5$; error bars represent mean \pm SEM. Kruskal-Wallis test with Dunn's multiple comparison was performed $(p$ value $=0.004$, Kruskal-Wallis statistic $=8.028$ for $\mathbf{A}),(p$ value $=0.0023$, Kruskal-Wallis statistic $=8.375$ for $\mathbf{B}$ )

purinergic communication. Interestingly, this effect was not $\mathrm{A}_{1}$ or $\mathrm{A}_{2 \mathrm{~A}} \mathrm{R}$ specific, as both compounds reverted the COderived effect. Also, treating microglia with the inactivated scaffold form of ALF-826 did not have an effect on either number of neurites per cell or neurite length (Fig. 8).

\section{Discussion}

In this study, we have shown that carbon monoxide (CO) modulates microglia function and impacts on microglia-toneuron remote communication. Using a conditioned media protocol, it was demonstrated that carbon monoxide ultimately promotes indirect neuroprotection: (i) by limiting microglial pro-inflammatory reactivity and (ii) by enhancing basal microglial neurotrophism. The non-cell autonomous effect of $\mathrm{CO}$ acting on microglia and promoting neuroprotection has never been described before. Furthermore, a novel molybdenum-based CO-releasing molecule: ALF-826 was used herein. This molecule presents low cytotoxicity in BV2 microglia cell line [56] and delivers a large CO load (personal information of Proterris (Portugal) Lda.).

The anti-inflammatory feature of $\mathrm{CO}$ has been extensively studied in macrophages [40,62]. Likewise, microglia treated with carbon monoxide-releasing molecule-3 (CORM-3) also secreted lower levels of tumour necrosis factor $\alpha$ (TNF- $\alpha$ ), interleukin-1 $\beta$ (IL-1 $\beta$ ) and nitrites after being triggered with different stimuli (lipopolysaccharide, thrombin and interferon $\gamma$ ) $[38,39]$. Although there are data demonstrating the 


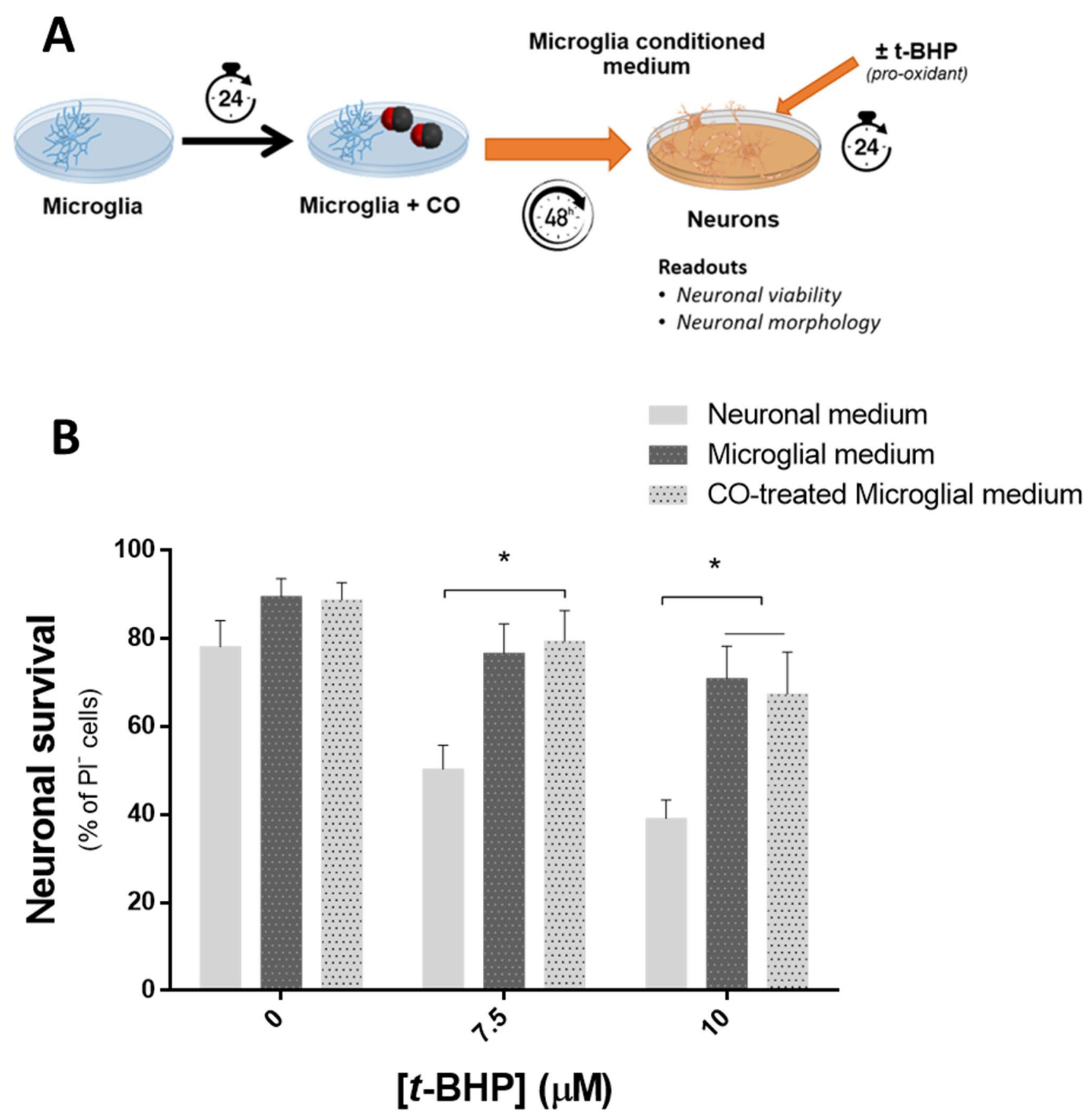

Fig. 5 Microglia-conditioned media provides a protective effect in neurons exposed to oxidative stress. BV2 microglia were treated with ALF-826 $(50 \mu \mathrm{M})$ for $48 \mathrm{~h}$. CAD neuronal culture was simultaneously incubated with microglia-conditioned medium and with pro-

anti-neuroinflammatory role of $\mathrm{CO}$ in microglia, not much is known regarding direct $\mathrm{CO}$ modulation of neuron-microglia communication in an inflammatory setting, and how it affects neuronal survival and function.

Herein, we demonstrated that conditioned media derived from microglia treated with a pro-inflammatory stimulus (LPS) promoted neuronal cell death. Neurons challenged oxidant agent $t$-BHP (A). Cell viability was assessed by propidium iodide staining quantification in flow cytometry $(\mathbf{B}) . n=6$; error bars represent mean \pm SEM; two-way ANOVA test with multiple comparisons was performed

with inflammatory microglial supernatant partially lost morphological complexity. Moreover, microglial pre-treatment with ALF-826 rescued changes in neuronal morphology and partially reverted neuronal death. Neuronal structural degradation is a marker of cell senescence and cell death in general and can be a consequence of exacerbated inflammation [63]. Alterations in neuronal network complexity are a 

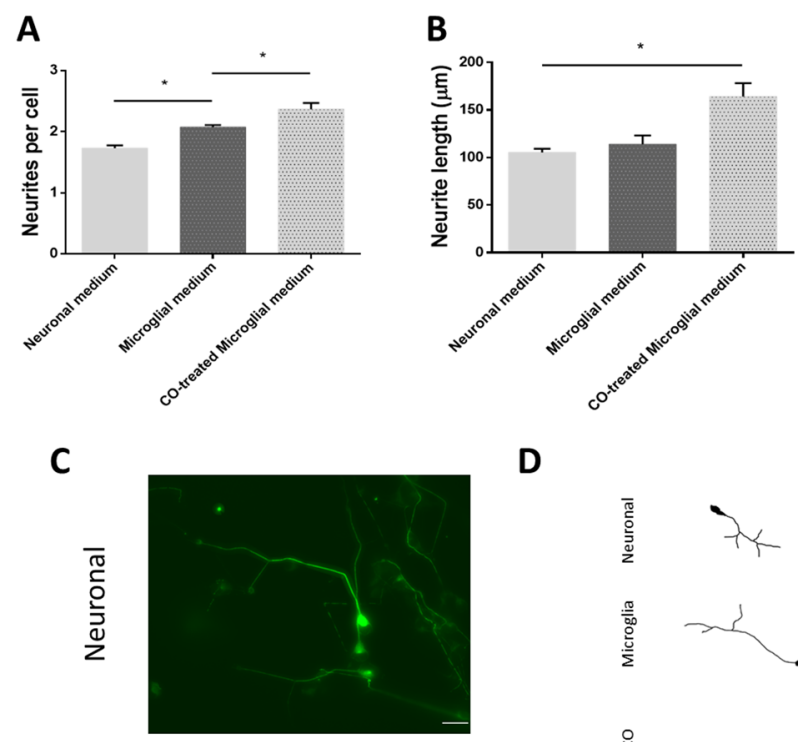

D
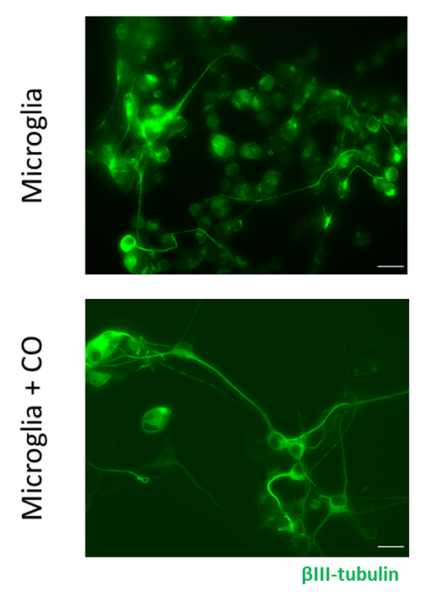

Fig. 6 Administration of $\mathrm{CO}$ in 'resting' microglia increases neuronal morphological complexity after exposure to conditioned medium. CAD neurons treated with BV2 microglia (previously treated or not with ALF-826 at $50 \mu \mathrm{M}$ ) conditioned media for $24 \mathrm{~h}$ were labelled with a $\beta$ III-tubulin antibody and DAPI, and fluorescence microscopy was performed. Micrographs were analysed, and morphological features, $\mathbf{A}$ number of neurites per cell and $\mathbf{B}$ neurite length, were assessed from five independent experiments (10-15 cells per condition). C Representative cell pictures for each condition with cells immunostained with $\beta$ III-tubulin primary antibody (scale bar $=25 \mu \mathrm{m}$ ) and $\mathbf{D}$ representative tracings of $2 \mathrm{D}$ acquired images. $n=5$; error bars represent mean \pm SEM. For $6 \mathbf{A}$, one-way ANOVA test with Tukey's multiple comparison test was performed ( $p$ value $<0.0001, F=22.94$ ), for $6 \mathbf{B}$, Kruskal-Wallis test with Dunn's multiple comparison was performed $(p$ value $=0.0327$, Kruskal-Wallis statistic $=6.269$ )

common phenotype of neurodegenerative disorders and play a role in defective synaptic connectivity [64-67]. It has been shown that exposure of primary embryonic neurons to high levels of exogenous TNF and IL- $1 \beta$ alters cell morphology, affecting total arborization, number and length of dendrites, growth cone dynamics as well as dendritic spine density and maturity [68]. Thus, loss of neuronal morphological complexity can also be partially a consequence of proinflammatory pathways disruption cytoskeleton-signalling mechanisms, which $\mathrm{CO}$ could alleviate by inhibiting microglial inflammatory output. These results indicate that LPS-treated microglia drive a noxious response that causes severe neuronal damage, which can be prevented by ALF826 microglial pre-treatment.

We assessed microglial production of soluble inflammatory factors in the presence and absence of ALF-826 administration. LPS increased secretion of soluble inflammatory factors TNF- $\alpha$ and nitrites, which were attenuated by $\mathrm{CO}$ administration in microglia. In contrast, LPS did not promote IL-1 $\beta$ release in BV2 microglia cell line (data not shown).

Despite microglia modulation of inflammation and CNS immunity, these myeloid cells also regulate brain development, synaptogenesis and neuronal turnover and provide strong neurotrophic support to neuronal cells $[12,19-24$, $57,58,69,70]$. Interestingly, CO modulation of microglial neurotrophic response has never been explored. Herein, we showed that conditioned media derived from non-activated microglia protected neurons from oxidative damage by partially preventing neuronal cell death. Microglia neuroprotection can result from secretion of neurotrophins (BDNF, neurotrophin-3, nerve growth factor) [20,71], inflammatory cytokines [24] or survival growth factors (IGF-1) [12], which impact cell fate. In the present work, $\mathrm{CO}$ did not increase microglial production and secretion of BDNF or GDNF, while IL-10 release was stimulated by CO. Microglial IL-10 is a key anti-inflammatory regulator, anti-apoptotic and an important synaptogenic mediator in vivo [72-74]. Accordingly, using in vivo acute inflammation model and in vitro LPS-stimulated macrophages, CO promoted IL-10 production [40]. Under physiological conditions without inflammatory stimulation, this is the first work showing CO-induced production of IL-10 in microglia. Furthermore, IL-10 promotes neurite outgrowth and synapse formation in OGD (oxygen-glucose deprivation)-challenged primary cortical neurons [60]. Herein, conditioned medium from CO-treated microglia increased number of neurites per cell and neurite length. Because IL-10 has a neuromorphogenic role [60], one may speculate that IL-10 is involved in neuronal morphology improvement by reinforcing neurotrophism.

Moreover, we unveiled that $\mathrm{CO}$ acts as a stimulator of microglia purinergic signalling, which affects neuronal function and survival. Purinergic signalling is important for cellular cross-talk and both microglial environmental sensing and reactivity [75], as well as neuronal survival and function [76]. Activation of adenosine receptor $\mathrm{A}_{2 \mathrm{~A}}$ has been reported to promote axonal elongation and dendritic branching in cortical neurons [77]. A recent breakthrough paper has shown that microglia can sense neuronal ATP, convert it into adenosine, which acts as 


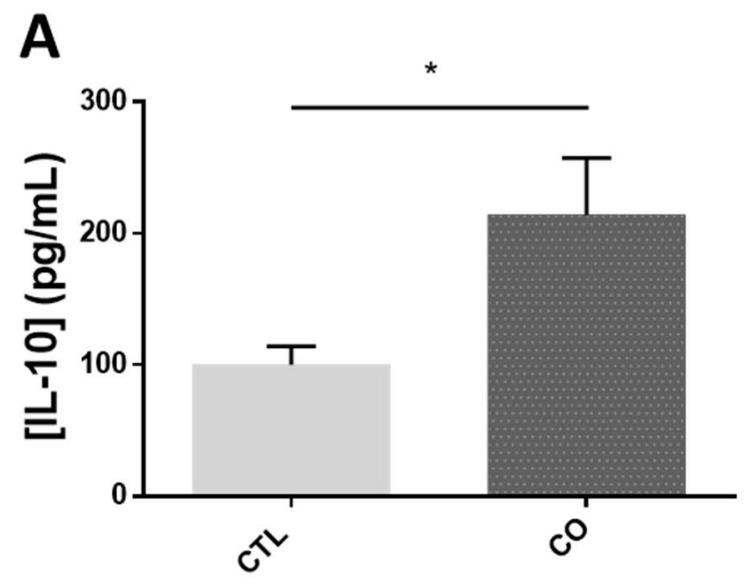

B
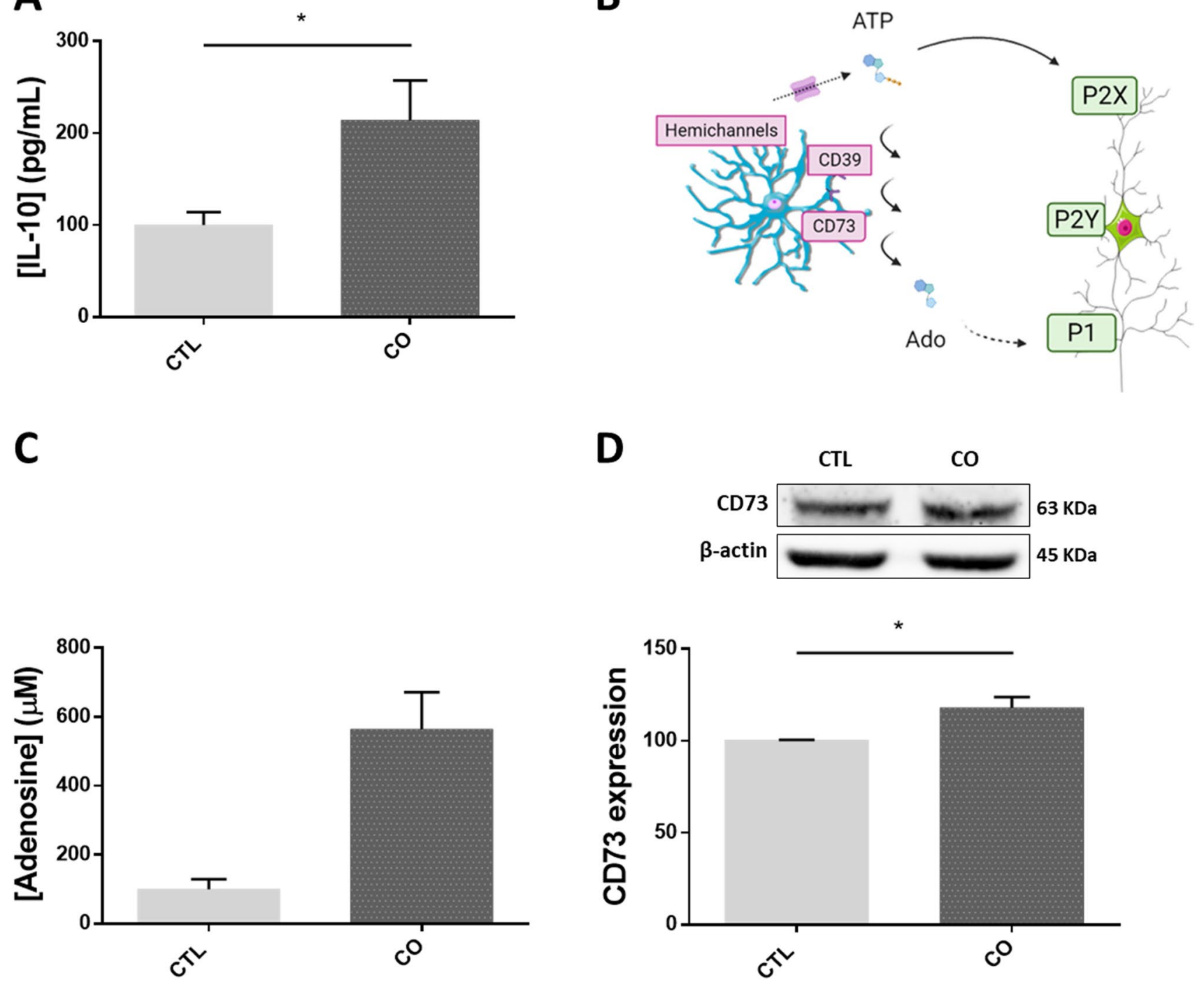

Fig. $7 \mathrm{CO}$ stimulates 'resting' microglial secretion of anti-inflammatory factor IL-10 and adenosine. BV2 microglia supernatant (derived from microglia treated or not with ALF-826 at $50 \mu \mathrm{M}$ ) was collected, and secretion levels of IL-10 was quantified by ELISA (A). Microglia communicate with neuronal cells via purinergic signalling (B). For the quantification of adenosine in the microglial media, the supernatant was collected, treated and analysed via HPLC (C). BV2 microglia CD73 protein expression was quantified by western blot-

ting (D). CD73 densitometry was normalised for $\beta$-actin. Results were normalised to total microglial protein levels. All results are presented as percentage relative to the negative control. $n=4-5$; error bars represent mean \pm SEM. For $7 \mathbf{A}$, unpaired $t$ test was performed ( $p$ value $=0.0377, t=2,486$ ), for 7C, Mann-Whitney $U$ test was performed ( $p$ value $=0.1$, Mann-Whitney $U=0.0$ ), for 7D Mann-Whitney test was performed $(p$ value $=0.0286$, Mann-Whitney $U=0.0$ )

an important suppressant of neuronal activity and overall animal behaviour [17]. We have previously shown similar results concerning astrocyte to neuron communication. Namely, CO-treated astrocytes provided neuroprotection and increased neuronal viability in a co-culture model by enhancing release and conversion of ATP into adenosine [61].

Interestingly, previous reports have also shown upregulation of ectonucleotidases following brain ischemia, providing cerebral protection [78]. In microglia, CD39 and

CD73 are crucial for microglial ramification, phagocytosis and motility [79]; and in vitro silencing of CD73 in BV2 microglia results in a clear pro-inflammatory profile [80]. Furthermore, CD73 expression is relevant for microglial polarisation and inflammatory attenuation in a spinal cord injury model [80]. This data could indicate that there is a paracrine feedback loop in microglia in which secreted ATP is converted into adenosine and recognised by the self, thus regulating microglial inflammatory activity. Herein, chemical inhibition of neuronal adenosine indicates that microglial 
A

Neuronal media

Microglia media

ALF-826

iALF-826

$\mathrm{SCH}-58261(\mu \mathrm{M})$

$\operatorname{DPCPX}(\mu \mathrm{M})$

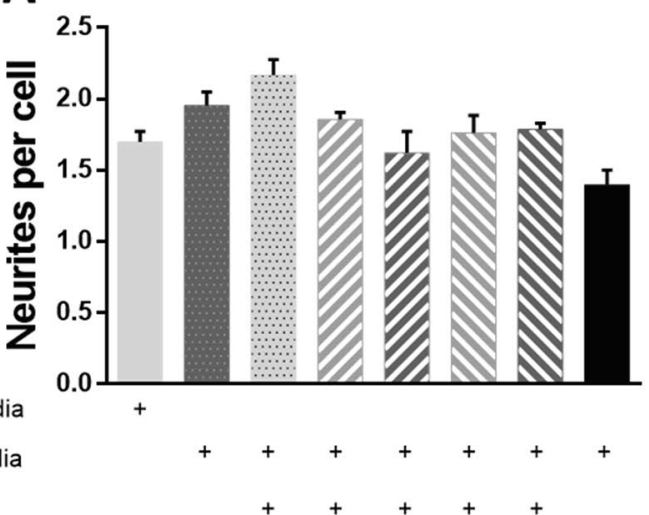

$+$

15

Fig. 8 Adenosine receptor blockade reverts neuronal morphological complexity improvement after exposure to CO-treated microgliaconditioned medium. CAD neurons treated with BV2 microglia-conditioned media and $\mathrm{P} 1$ receptor inhibitors $(\mathrm{SCH}-58261,1$ and $5 \mu \mathrm{M}$ and DPCPX, 25 and $50 \mu \mathrm{M}$ ) for $24 \mathrm{~h}$ were labelled with a $\beta$ III-tubulin antibody and DAPI, and fluorescence microscopy was performed. Micrographs were analysed and morphological features, (A) number

adenosine has a direct effect on neuronal function, but we cannot exclude that $\mathrm{CO}$ also regulates microglial CD73 expression, increasing adenosine levels in the supernatant, priming microglia to release higher levels of other neuroactive compounds. This would be in accordance with existing results, that claim that $\mathrm{CD} 73$ activity drives microglia towards an $\mathrm{M} 2$ profile by autocrine $\mathrm{A}_{2 \mathrm{~B}}$ agonism. Further experiments are needed to assess this hypothesis.

In summary, neurotrophic factors, anti-oxidant molecules or even anti-apoptotic players can be involved in microglia-induced neuroprotection under non-inflammatory conditions.

\section{Conclusion}

Taken together, ALF-826 is a promising new CO releasing molecule that limits microglial reactivity and secretion of inflammatory mediators, and that improves the release of neurotrophic factors. Both effects protect neurons against cell death and improve neuronal morphology. In fact and to the best of our knowledge, this is the first report showing that $\mathrm{CO}$ affects basal microglia function, by stimulating neurotrophism. Hence, $\mathrm{CO}$ regulates neuron-microglia remote communication and promotes neuroprotection in a non-cell autonomous manner by both limiting inflammation and improving neurotrophism. The novel ALF-826 has also proven to be a useful CORM for the reproducible and

B

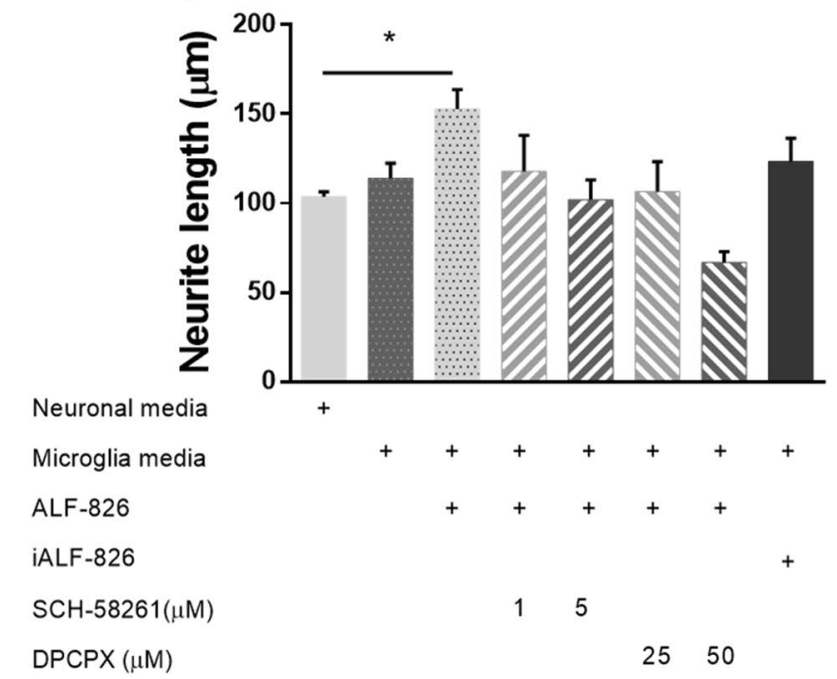

of neurites per cell and (B) neurite length, were assessed from four independent experiments (15-20 cells per condition). $n=4-8$; error bars represent mean \pm SEM. For 8 A, Kruskal-Wallis test with Dunn's multiple comparison was performed $(p$ value $=0.014$, Kruskal-Wallis statistic $=17.57$ ), for $8 \mathbf{B}$, one-way ANOVA test with Tukey's multiple comparison test was performed $(p$ value $=0.0008, F=4.582$ )

non-toxic delivery of $\mathrm{CO}$ enabling the study of intercellular processes and with potential for future clinical applications.

Supplementary Information The online version contains supplementary material available at https://doi.org/10.1007/s12035-021-02643-z.

Author Contribution NLS designed the study, conducted the experiments, helped with data analysis and evaluation, wrote the manuscript and approved the final version of this manuscript. IP conducted the experiments, helped with data analysis and approved the final version of this manuscript. JB helped with primary cultures and approved the final version of this manuscript. CSFQ designed part of the study, conducted some of the experiments and approved the final version of this manuscript. BFM and SVC helped with HPLC experiments and data analysis and approved the final version of this manuscript. CCR provided the CORM ALF-826 (which is not available commercially), helped with data analysis and approved the final version of this manuscript. TS designed part of the study, helped with data analysis and approved the final version of this manuscript. HLAV designed the study, helped with data analysis and evaluation, wrote the manuscript and approved the final version of this manuscript.

Funding This work was financed by FEDER-Fundo Europeu de Desenvolvimento Regional funds through the COMPETE 2020-Operational Programme for Competitiveness and Internationalization (POCI), Portugal 2020, and by Portuguese funds through FCT-Fundação para a Ciência e a Tecnologia/Ministério da Ciência (FCT), Tecnologia e Ensino Superior in the framework of FCT-ANR/NEUNMC/0022/2012 grant, PTDC/MEC-NEU/28750/2017 grant, Applied Molecular Biosciences Unit-UCIBIO (UID/Multi/04378/2019) grant; iNOVA4Health-Programme in Translational Medicine (UID/ Multi/04462/2013) grant and LA/P/0140/2020 of the Associate Laboratory Institute for Health and Bioeconomy; and FCT provided 
individual financial support to NLS (PD/BD/127819/2016), BFM (PD/ BD/128336/2017) and HLAV (IF/00185/2012).

Data Availability The datasets generated during and/or analysed during the current study are available from the corresponding author on a reasonable request.

\section{Declarations}

Ethics Approval and Consent to Participate All animal experiments were performed with the approval of the i3S Animal Ethics Committee and in accordance with European Union Directive on the protection of animals used for scientific purposes (2010/63/EU) and the Portuguese Law that transposes it (Decreto-Lei No. 113/2013). All efforts were made to minimise animal suffering.

Consent for Publication All authors agreed to the publication of this manuscript.

Competing Interests The co-author Carlos C. Romão is a scientific consultant of Proterris (Portugal) Lda and has an equity position in the company.

\section{References}

1. Kettenmann H, Hanisch U-K, Noda M, Verkhratsky A (2011) Physiology of microglia. Physiol Rev 91:461-553. https://doi.org/ 10.1152/physrev.00011.2010

2. Ginhoux F, Greter M, Leboeuf M et al (2010) Fate Mapping Analysis Reveals That Adult Microglia Derive from Primitive Macrophages. Science (80-) 330:841-845. https://doi.org/10. 1126/science. 1194637

3. Salter MW, Stevens B (2017) Microglia emerge as central players in brain disease. Nat Med 23:1018-1027. https://doi.org/10.1038/ nm.4397

4. Colton CA, Gilbert DL (1987) Production of superoxide anions by a CNS macrophage, the microglia. FEBS Lett 223:284-288

5. Infanger DW, Sharma RV, Davisson RL (2006) NADPH Oxidases of the Brain: Distribution, Regulation, and Function. Antioxid Redox Signal 8:1583-1596. https://doi.org/10.1089/ars.2006.8. 1583

6. Neniskyte U, Vilalta A, Brown GC (2014) Tumour necrosis factor alpha-induced neuronal loss is mediated by microglial phagocytosis. FEBS Lett 588:2952-2956. https://doi.org/10.1016/j.febsl et.2014.05.046

7. Sedel F, Béchade C, Vyas S, Triller A (2004) MacrophageDerived Tumor Necrosis Factor $\alpha$, an Early Developmental Signal for Motoneuron Death. J Neurosci. https://doi.org/10.1523/ JNEUROSCI.4464-03.2004

8. Paolicelli RC, Bergamini G, Rajendran L (2019) Cell-to-cell Communication by Extracellular Vesicles: Focus on Microglia. Neuroscience 405:148-157

9. Neumann H, Kotter MR, Franklin RJM (2009) Debris clearance by microglia: An essential link between degeneration and regeneration. Brain 132:288-295

10. Neher JJ, Emmrich JV, Fricker M et al (2013) Phagocytosis executes delayed neuronal death after focal brain ischemia. Proc Natl Acad Sci 110:E4098-E4107. https://doi.org/10.1073/pnas. 1308679110

11. Ellwardt E, Walsh JT, Kipnis J, Zipp F (2016) Understanding the Role of T Cells in CNS Homeostasis. Trends Immunol $37: 154-165$
12. Arnoux I, Hoshiko M, Sanz Diez A, Audinat E (2014) Paradoxical effects of minocycline in the developing mouse somatosensory cortex. Glia. https://doi.org/10.1002/glia.22612

13. Mosser CA, Baptista S, Arnoux I, Audinat E (2017) Microglia in CNS development: Shaping the brain for the future. Prog Neurobiol 149-150:1-20. https://doi.org/10.1016/j.pneurobio. 2017.01.002

14. Paolicelli RC, Bolasco G, Pagani F, et al (2011) Synaptic pruning by microglia is necessary for normal brain development. Science (80-). https://doi.org/10.1126/science.1202529

15. Schafer DP, Lehrman EK, Kautzman AG et al (2012) Microglia Sculpt Postnatal Neural Circuits in an Activity and Complement-Dependent Manner. Neuron. https://doi.org/10.1016/j. neuron.2012.03.026

16. Tremblay MĚ, Lowery RL, Majewska AK (2010) Microglial interactions with synapses are modulated by visual experience. PLoS Biol. https://doi.org/10.1371/journal.pbio.1000527

17. Badimon A, Strasburger HJ, Ayata P, et al (2020) Negative feedback control of neuronal activity by microglia. Nature 586 . https://doi.org/10.1038/s41586-020-2777-8

18. Miyamoto A, Wake H, Ishikawa AW et al (2016) Microglia contact induces synapse formation in developing somatosensory cortex. Nat Commun. https://doi.org/10.1038/ncomms12540

19. Tremblay MÈ, Stevens B, Sierra A et al (2011) The role of microglia in the healthy brain. J Neurosci. https://doi.org/10. 1523/JNEUROSCI.4158-11.2011

20. Morgan SC, Taylor DL, Pocock JM (2004) Microglia release activators of neuronal proliferation mediated by activation of mitogen-activated protein kinase, phosphatidylinositol-3-kinase/ Akt and delta-Notch signalling cascades. J Neurochem 90:89-101

21. Nagata K, Takei N, Nakajima K et al (1993) Microglial conditioned medium promotes survival and development of cultured mesencephalic neurons from embryonic rat brain. J Neurosci Res. https://doi.org/10.1002/jnr.490340313

22. Chamak B, Dobbertin A, Mallat M (1995) Immunohistochemical detection of thrombospondin in microglia in the developing rat brain. Neuroscience. https://doi.org/10.1016/0306-4522(95) 00236-C

23. Arnoux I, Hoshiko M, Mandavy L et al (2013) Adaptive phenotype of microglial cells during the normal postnatal development of the somatosensory "Barrel" cortex. Glia. https://doi. org/10.1002/glia.22503

24. Shigemoto-Mogami Y, Hoshikawa K, Goldman JE et al (2014) Microglia enhance neurogenesis and oligodendrogenesis in the early postnatal subventricular zone. J Neurosci. https://doi.org/ 10.1523/JNEUROSCI.1619-13.2014

25. Parkhurst CN, Yang G, Ninan I et al (2013) Microglia promote learning-dependent synapse formation through brain-derived neurotrophic factor. Cell. https://doi.org/10.1016/j.cell.2013.11. 030

26. Lim SH, Park E, You B et al (2013) Neuronal synapse formation induced by microglia and interleukin 10. PLoS ONE. https://doi. org/10.1371/journal.pone.0081218

27. Pascual O, Ben AS, Rostaing P et al (2012) Microglia activation triggers astrocyte-mediated modulation of excitatory neurotransmission. Proc Natl Acad Sci U S A. https://doi.org/10.1073/pnas. 1111098109

28. Poon VY, Choi S, Park M (2013) Growth factors in synaptic function. Front. Synaptic Neurosci. http://doi.org/10.3389/fnsyn.2013. 00006

29. Heneka MTM, Carson MJ, El KJEJ et al (2015) Neuroinflammation in Alzheimer's disease. Lancet Neurol. https://doi.org/10. 1016/S1474-4422(15)70016-5

30. Hu X, Li P, Guo Y et al (2012) Microglia/macrophage polarization dynamics reveal novel mechanism of injury expansion after focal 
cerebral ischemia. Stroke 43:3063-3070. https://doi.org/10.1161/ STROKEAHA.112.659656

31. Tenhunen R, Marver HS, Schmid R (1968) The enzymatic conversion of heme to bilirubin by microsomal heme oxygenase. Proc Natl Acad Sci 61:748-755. https://doi.org/10.1073/pnas.61.2.748

32. Ryter SW, Alam J, Choi AMK (2006) Heme Oxygenase-1/Carbon Monoxide: From Basic Science to Therapeutic Applications. Physiol Rev 86:583-650. https://doi.org/10.1152/physrev.00011. 2005

33. Ryter SW, Choi AMK (2016) Targeting heme oxygenase-1 and carbon monoxide for therapeutic modulation of inflammation. Transl Res 167:7-34

34. Figueiredo-Pereira C, Dias-Pedroso D, Soares NL, Vieira HLA (2020) CO-mediated cytoprotection is dependent on cell metabolism modulation. Redox Biol. 32. https://doi.org/10.1016/j.redox. 2020.101470

35. Queiroga CSF, Vercelli A, Vieira HLA (2015) Carbon monoxide and the CNS: challenges and achievements. Br J Pharmacol 172:1533-1545. https://doi.org/10.1111/bph.12729

36. Bilban M, Bach FH, Otterbein SL et al (2006) Carbon Monoxide Orchestrates a Protective Response through PPAR $\gamma$. Immunity 24:601-610. https://doi.org/10.1016/j.immuni.2006.03.012

37. Hoetzel A, Dolinay T, Vallbracht $\mathrm{S}$ et al (2008) Carbon monoxide protects against ventilator-induced lung injury via PPAR- $\gamma$ and inhibition of Egr-1. Am J Respir Crit Care Med 177:1223-1232. https://doi.org/10.1164/rccm.200708-1265OC

38. Bani-Hani MG, Greenstein D, Mann BE et al (2006) Modulation of thrombin-induced neuroinflammation in BV-2 microglia by carbon monoxide-releasing molecule 3. J Pharmacol Exp Ther 318:1315-1322. https://doi.org/10.1124/jpet.106.104729

39. Peyton KJ, Reyna SV, Chapman GB et al (2002) Heme oxygenase-1 - derived carbon monoxide is an autocrine inhibitor of vascular smooth muscle cell growth. Blood 99:4443-4448

40. Otterbein LE, Bach FH, Alam J et al (2000) Carbon monoxide has anti-inflammatory effects involving the mitogen-activated protein kinase pathway. Nat Med 6:422-428. https://doi.org/10. $1038 / 74680$

41. Vieira HLA, Queiroga CSF, Alves PM (2008) Pre-conditioning induced by carbon monoxide provides neuronal protection against apoptosis. J Neurochem 107:375-384. https://doi.org/10.1111/j. 1471-4159.2008.05610.x

42. Schallner N, Romão CC, Biermann J, et al (2013) Carbon Monoxide Abrogates Ischemic Insult to Neuronal Cells via the Soluble Guanylate Cyclase-cGMP Pathway. PLoS One 8. https://doi.org/ 10.1371/journal.pone.0060672

43. Wang B, Cao W, Biswal S, Doré S (2011) Carbon monoxide-activated Nrf2 pathway leads to protection against permanent focal cerebral ischemia. Stroke 42:2605-2610. https://doi.org/10.1161/ STROKEAHA.110.607101

44. Queiroga CSF, Tomasi S, Widerøe M, et al (2012) Preconditioning Triggered by Carbon Monoxide (CO) Provides Neuronal Protection Following Perinatal Hypoxia-Ischemia. PLoS One 7. https:// doi.org/10.1371/journal.pone.0042632

45. Queiroga CSF, Almeida AS, Martel C et al (2010) Glutathionylation of Adenine Nucleotide Translocase Induced by Carbon Monoxide Prevents Mitochondrial Membrane Permeabilization and Apoptosis. J Biol Chem 285:17077-17088. https://doi.org/ 10.1074/jbc.M109.065052

46. Almeida AS, Queiroga CSF, Sousa MFQ et al (2012) Carbon monoxide modulates apoptosis by reinforcing oxidative metabolism in astrocytes: Role of Bcl-2. J Biol Chem 287:10761-10770. https://doi.org/10.1074/jbc.M111.306738

47. Oliveira SR, Figueiredo-Pereira C, Duarte CB, Vieira HLA (2019) P2X7 Receptors Mediate CO-Induced Alterations in Gene Expression in Cultured Cortical
Astrocytes-Transcriptomic Study. Mol Neurobiol 56:31593174. https://doi.org/10.1007/s12035-018-1302-7

48. Parfenova H, Tcheranova D, Basuroy S et al (2012) Functional role of astrocyte glutamate receptors and carbon monoxide in cerebral vasodilation response to glutamate. AJP Hear Circ Physiol 302:H2257-H2266. https://doi.org/10.1152/ajpheart. 01011.2011

49. Bani-Hani MG, Greenstein D, Mann BE et al (2006) A carbon monoxide-releasing molecule (CORM-3) attenuates lipopolvsaccharide- And interferon- $\gamma$-induced inflammation in microglia. Pharmacol Reports 58:132-144

50. Wilson JLJL, Bouillaud F, Almeida ASAS et al (2017) Carbon monoxide reverses the metabolic adaptation of microglia cells to an inflammatory stimulus. Free Radic Biol Med 104:311323. https://doi.org/10.1016/j.freeradbiomed.2017.01.022

51. Chora AA, Fontoura P, Cunha A et al (2007) Heme oxygenase-1 and carbon monoxide suppress autoimmune neuroinflammation. J Clin Invest 117:438-447. https://doi.org/10.1172/JCI28844

52. Yabluchanskiy A, Sawle P, Homer-Vanniasinkam S et al (2012) CORM-3, a carbon monoxide-releasing molecule, alters the inflammatory response and reduces brain damage in a rat model of hemorrhagic stroke. Crit Care Med 40:544-552. https://doi. org/10.1097/CCM.0b013e31822f0d64

53. Motterlini R, Clark JE, Foresti R et al (2002) Carbon monoxidereleasing molecules: characterization of biochemical and vascular activities. Circ Res 90:e17-e24. https://doi.org/10.1161/ hh0202.104530

54. Johnson TR, Mann BE, Clark JE, et al (2003) Metal carbonyls: A new class of pharmaceuticals? Angew. Chemie - Int. Ed. 42:3722-3729

55. Motterlini R, Sawle P, Hammad J et al (2005) CORM-A1: a new pharmacologically active carbon monoxide-releasing molecule. Faseb J 19:284-286. https://doi.org/10.1096/fj.04-2169fje

56. Dias-Pedroso D, Ramalho J-S, Sardão V., et al (2021) Carbon monoxide-Neuroglobin axis targeting metabolism against inflammation in BV-2 microglial cells. Mol Neurobiol. http:// doi.org/10.1007/s12035-021-02630-4

57. Kettenmann H, Kirchhoff F, Verkhratsky A (2013) Microglia: New Roles for the Synaptic Stripper. Neuron 77:10-18

58. Frost JL, Schafer DP (2016) Microglia: Architects of the Developing Nervous System. Trends Cell Biol. 26:587-597

59. Pérez-De Puig I, Miró F, Salas-Perdomo A, et al (2013) IL-10 deficiency exacerbates the brain inflammatory response to permanent ischemia without preventing resolution of the lesion. J Cereb Blood Flow Metab 33. https://doi.org/10.1038/jcbfm. 2013.155

60. Chen H, Lin W, Zhang Y, et al (2016) IL-10 Promotes Neurite Outgrowth and Synapse Formation in Cultured Cortical Neurons after the Oxygen-Glucose Deprivation via JAK1/STAT3 Pathway. Sci Rep 6. https://doi.org/10.1038/srep30459

61. Queiroga CSFCSFCSF, Alves RMARMARMA, Conde SVSVSV et al (2016) Paracrine effect of carbon monoxide: astrocytes promote neuroprotection via purinergic signaling. J Cell Sci 129:3178-3188. https://doi.org/10.1242/jcs. 187260

62. Sawle P, Foresti R, Mann BE et al (2005) Carbon monoxidereleasing molecules (CO-RMs) attenuate the inflammatory response elicited by lipopolysaccharide in RAW264.7 murine macrophages. Br J Pharmacol 145:800-810. https://doi.org/10. 1038/sj.bjp.0706241

63. Galluzzi L, Vitale I, Aaronson SA, et al (2018) Molecular mechanisms of cell death: Recommendations of the Nomenclature Committee on Cell Death 2018. Cell Death Differ. 25, 3. http://doi.org/ 10.1038/s41418-017-0012-4

64. Yuan TF, Peng B, Machado S, Arias-Carrion O (2015) Morphological Bases of Neuronal Hyperexcitability in Neurodegeneration. CNS Neurosci. Ther. 21:867-869 
65. Ferrer I (1999) Neurons and their dendrites in frontotemporal dementia. Dement Geriatr Cogn Disord 10. https://doi.org/10. $1159 / 000051214$

66. Spires TL, Hyman BT (2004) Neuronal structure is altered by amyloid plaques. Rev. Neurosci. 15:267-278

67. Šišková Z, Justus D, Kaneko H, et al (2014) Dendritic structural degeneration is functionally linked to cellular hyperexcitability in a mouse model of alzheimer's disease. Neuron 84. https://doi.org/ 10.1016/j.neuron.2014.10.024

68. Frias, Cátia; Brites, Dora; Fernandes A (2011) Master thesis: Dissecting neuronal development deficits by inflammation : from morphology to cytoskeleton dynamics. Faculdade de Farmácia da Universidade de Lisboa. http://hdl.handle.net/10362/6710

69. Antony JM, Paquin A, Nutt SL et al (2011) Endogenous microglia regulate development of embryonic cortical precursor cells. J Neurosci Res. https://doi.org/10.1002/jnr.22533

70. Cunningham CL, Martínez-Cerdeño V, Noctor SC (2013) Microglia regulate the number of neural precursor cells in the developing cerebral cortex. J Neurosci. https://doi.org/10.1523/JNEUR OSCI.3441-12.2013

71. Frade JM, Barde YA (1998) Microglia-derived nerve growth factor causes cell death in the developing retina. Neuron 20. https:// doi.org/10.1016/S0896-6273(00)80432-8

72. Bachis A, Colangelo AM, Vicini S, et al (2001) Interleukin-10 prevents glutamate-mediated cerebellar granule cell death by blocking caspase-3-like activity. J Neurosci 21. https://doi.org/ 10.1523/jneurosci.21-09-03104.2001

73. Sawada M, Suzumura A, Hosoya H, et al (1999) Interleukin-10 inhibits both production of cytokines and expression of cytokine receptors in microglia. J Neurochem 72. https://doi.org/10.1046/j. 1471-4159.1999.721466.x
74. Lodge PA, Sriram S (1996) Regulation of microglial activation by TGF- $\beta$, IL-10, and CSF-1. J Leukoc Biol 60. https://doi.org/ 10.1002/jlb.60.4.502

75. Illes P, Rubini P, Ulrich H, et al (2020) Regulation of Microglial Functions by Purinergic Mechanisms in the Healthy and Diseased CNS. Cells 9:5. http://doi.org/10.3390/cells9051108

76. Fields RD, Burnstock G (2006) Purinergic signalling in neuronglia interactions. Nat Rev Neurosci 7:423-436

77. Assaife-Lopes N, Sousa VC, Pereira DB, et al (2010) Activation of adenosine $\mathrm{A} 2 \mathrm{~A}$ receptors induces TrkB translocation and increases BDNF-mediated phospho-TrkB localization in lipid rafts: Implications for neuromodulation. J Neurosci 30. https:// doi.org/10.1523/JNEUROSCI.5695-09.2010

78. Shinozaki Y, Koizumi S, Ishida S et al (2005) Cytoprotection against oxidative stress-induced damage of astrocytes by extracellular ATP via P2Y1 receptors. Glia 49:288-300

79. Matyash M, Zabiegalov O, Wendt S, et al (2017) The adenosine generating enzymes CD39/CD73 control microglial processes ramification in the mouse brain. PLoS One 12. https://doi.org/10. 1371/journal.pone.0175012

80. Xu S, Zhu W, Shao M, et al (2018) Ecto-5'-nucleotidase (CD73) attenuates inflammation after spinal cord injury by promoting macrophages/microglia M2 polarization in mice. J Neuroinflammation 15. https://doi.org/10.1186/s12974-018-1183-8

Publisher's Note Springer Nature remains neutral with regard to jurisdictional claims in published maps and institutional affiliations. 